TRANSACTIONS OF THE

AMERICAN MATHEMATICAL SOCIETY

Volume 361, Number 1, January 2009, Pages 237-265

S 0002-9947(08)04568-6

Article electronically published on August 13, 2008

\title{
TOWARDS INVARIANTS OF SURFACES IN 4-SPACE VIA CLASSICAL LINK INVARIANTS
}

\author{
SANG YOUL LEE
}

\begin{abstract}
In this paper, we introduce a method to construct ambient isotopy invariants for smooth imbeddings of closed surfaces into 4-space by using hyperbolic splittings of the imbedded surfaces and an arbitrary given isotopy or regular isotopy invariant of classical knots and links in 3-space. Using this construction, adopting the Kauffman bracket polynomial as an example, we produce some invariants.
\end{abstract}

\section{INTRODUCTION}

By a surface link (or knotted surface) of $n$ components we mean a locally flat closed (possibly disconnected or non-orientable) surface $\mathcal{L}=F_{1} \cup F_{2} \cup \cdots \cup F_{n}, n \geq$ 1 , imbedded in the oriented 4-dimensional Euclidean space $\mathbb{R}^{4}$ (or the oriented 4-sphere $S^{4}$ ), where each component $F_{i}$ is homeomorphic to a closed connected surface. A surface link with one component is sometimes called a surface knot. If $F_{i}$ is homeomorphic to the 2 -sphere $S^{2}$, then $\mathcal{L}$ is called a 2 -link of $n$-components. In particular, a 2-link with one component is called a 2-knot. If each component $F_{i}$ is oriented, then we call $\mathcal{L}$ an oriented surface link. Throughout this paper, we work in the piecewise linear or smooth category. Two surface links $\mathcal{L}$ and $\mathcal{L}^{\prime}$ in $\mathbb{R}^{4}$ are said to be equivalent or of the same link type if there exists an orientation preserving homeomorphism $\Phi: \mathbb{R}^{4} \rightarrow \mathbb{R}^{4}$ such that $\Phi(\mathcal{L})=\mathcal{L}^{\prime}$. If $\mathcal{L}$ and $\mathcal{L}^{\prime}$ are oriented surface links, then it is assumed that the restriction $\left.\Phi\right|_{\mathcal{L}}: \mathcal{L} \rightarrow \mathcal{L}^{\prime}$ is an orientation preserving homeomorphism.

One of the most popular diagrammatic methods to describe surface links in $\mathbb{R}^{4}$ is one based on generic projections of surface links into $\mathbb{R}^{3}$ and associated Roseman's moves for surface isotopies [5, 23. The other is knots with bands derived from normal forms of surface links in $\mathbb{R}^{4}[8,11,14,15,22$. The braid theory in dimension 4 is also such a method $9,10,11,24$. These approaches give rise to the rich theory of algebraic and categorical aspects which produce many useful invariants for surface links in $\mathbb{R}^{4}$, for example 2, 3, 5, 9, 19, and therein.

Regular projection of the intersection of a hyperbolic splitting of a surface link in $\mathbb{R}^{4}$ and the 0-level cross section $\mathbb{R}^{3} \times\{0\}$ into the plane $\mathbb{R}^{2}$ gives a 4-regular spatial graph diagram in $\mathbb{R}^{2}$. Imposing an extra structure, a marker, for each 4valent vertex which indicates how the saddle point opens up above with respect to

Received by the editors December 18, 2006.

2000 Mathematics Subject Classification. Primary 57Q45; Secondary 57M25.

Key words and phrases. Kauffman bracket polynomial, knotted surface, knot with bands, surface link, Yoshikawa moves, ch-diagram.

(C)2008 American Mathematical Society Reverts to public domain 28 years from publication 
the height function, we have a planar diagram representing the surface link, called a ch-diagram of the surface link; cf. [1, 25]. In [27, K. Yoshikawa introduced planar local moves $\Omega_{1}, \ldots, \Omega_{8}$ on these marked 4 -valent spatial graph diagrams, which can be realized as ambient isotopies of surface links in $\mathbb{R}^{4}$ (see Figures 3 and (4). Two surface links $\mathcal{L}$ and $\mathcal{L}^{\prime}$ are said to be stably equivalent if their ch-diagrams are transformed into each other by a finite sequence of these moves, including their mirror moves. It was conjectured 27] that two surface links $\mathcal{L}$ and $\mathcal{L}^{\prime}$ are equivalent if and only if they are stably equivalent. In 2001, F. J. Swenton [26] claimed to have proved that this Yoshikawa conjecture is true. It should be noticed that a gap has been found in Swenton's paper, and it is recovered by S. Kamada.

On the other hand, the author [16 constructed the invariants for Yoshikawa's moves by using a state-sum model similar to Kauffman's state-sum model 13 . for the Jones polynomial for classical knots and links in $\mathbb{R}^{3}$. The purpose of this paper is to view the construction from a more general perspective and develop a more general framework to construct invariants of equivalent surface links by using invariants for classical knots and links in 3-space. This paper is the first in a series of works on the study of surface links via classical link invariants. In the second 17 . we examine our method developed through this paper with an elementary classical link invariant, the number of components, and the derived invariants are discussed. In the third [18 we also examine the method with invariants for magnetic graphs [20, 21] and produce invariants for oriented surface links in 4-space.

In this paper, for any given ambient isotopy or regular isotopy invariant of (unoriented) classical knots and links in 3 -space $\mathbb{R}^{3}$ or $S^{3}$ with its values in a commutative ring $R$ and a hyperbolic splitting $D$ of a surface link $\mathcal{L}$ in $\mathbb{R}^{4}$, we first define a polynomial $[[D]]$ in four variables with coefficients in $R$ via a skein relation and give its state-sum model and some properties. Then we describe a method to construct invariants of equivalent surface links by modifying this polynomial $[[D]]$. As an application, we examine this method with the Kauffman bracket polynomial for classical knot and link diagrams and investigate the produced invariants of surface links in $\mathbb{R}^{4}$.

This paper is organized as follows. In Section 2, the basic terminologies of hyperbolic splitting of surface links and Yoshikawa moves are briefly reviewed. In Section 3, we define the polynomial $[[D]]$ for marked 4-valent spatial graph diagrams and discuss its properties. In Section 4, we present our method to construct invariants of surface links. In Section 5, we investigate the invariants obtained from the method with the Kauffman bracket polynomial.

\section{Hyperbolic SPlitTing of SURfaCe LinkS IN $\mathbb{R}^{4}$}

We denote the hyperplane of $\mathbb{R}^{4}$ whose fourth coordinate $x_{4}$ is equal to $t \in$ $\mathbb{R}$ by $\mathbb{R}_{t}^{3}$; that is, $\mathbb{R}_{t}^{3}=\left\{\left(x_{1}, x_{2}, x_{3}, x_{4}\right) \in \mathbb{R}^{4} \mid x_{4}=t\right\}$. It is well known (cf. [6, 7, 8, 11, 14, 15, 19]) that for any surface link $\mathcal{L}$ in $\mathbb{R}^{4}$, there exists a surface link $\tilde{\mathcal{L}}$ in $\mathbb{R}^{4}$ satisfying the following conditions:

- $\tilde{\mathcal{L}}$ is equivalent to $\mathcal{L}$ and has only finitely many critical points, all of which are elementary.

- All maximal points of $\tilde{\mathcal{L}}$ are in the hyperplane $\mathbb{R}_{1}^{3}$.

- All minimal points of $\tilde{\mathcal{L}}$ are in the hyperplane $\mathbb{R}_{-1}^{3}$.

- All saddle points of $\tilde{\mathcal{L}}$ are in the hyperplane $\mathbb{R}_{0}^{3}$. 
Such a representation $\tilde{\mathcal{L}}$ is called a hyperbolic splitting of $\mathcal{L}$ [19]. Suppose that a surface link $\mathcal{L}$ in $\mathbb{R}^{4}$ is described by a hyperbolic splitting $\tilde{\mathcal{L}}$. Then the intersection $\tilde{\mathcal{L}} \cap \mathbb{R}_{0}^{3}$ of such a surface $\tilde{\mathcal{L}}$ with the 0 -level cross section $\mathbb{R}_{0}^{3}$ is a 4 -valent spatial graph in the 3-space $\mathbb{R}_{0}^{3}$. Imposing an extra structure, "marker", for each vertex, that is, for each saddle point, we indicate how the saddle points open up above, as shown in Figure 1

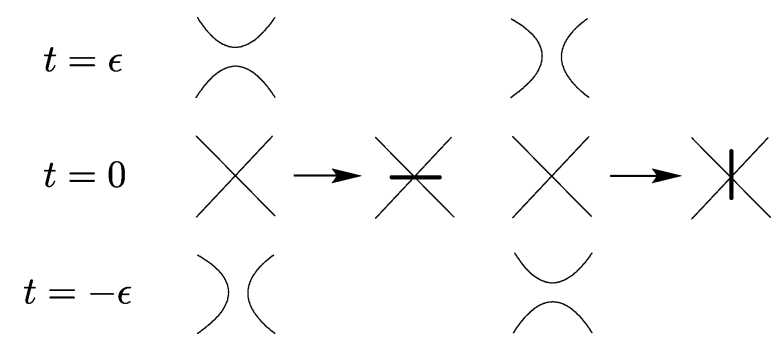

FiguRe 1. Marking of a vertex

As usual we describe such a marked 4-valent spatial graph in $\mathbb{R}_{0}^{3} \cong \mathbb{R}^{3}$ by its regular projection on the plane $\mathbb{R}^{2}$ with over and under crossings indicated in the standard way and with marked vertices, called a ch-diagram of the surface link $\mathcal{L}$. In what follows we denote the set of all classical crossings and marked vertices in a ch-diagram $D$ by $C(D)$ and $V(D)$, respectively, and the number of all classical crossings and vertices of $D$ are denoted by $|C(D)|$ and $|V(D)|$, respectively.

Let $D$ be a 4 -valent spatial graph diagram in $\mathbb{R}^{2}$ with marked vertices. Define $L_{+}(D)$ and $L_{-}(D)$ to be the classical link diagrams obtained from $D$ by replacing each marked vertex, as illustrated in Figure 2,

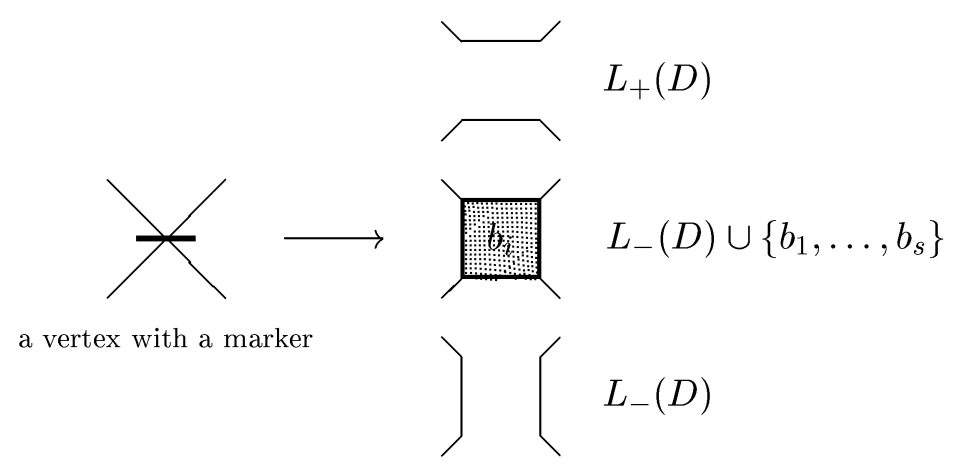

Figure 2

If $L_{+}(D)$ and $L_{-}(D)$ are diagrams which both represent trivial links, then we can define a surface link in $\mathbb{R}^{4}$ associated with $D$.

Theorem 2.1 ([14, 27]). Any surface link in $\mathbb{R}^{4}$ is represented by some ch-diagram.

On the other hand, let $\mathcal{L}$ be a surface link in $\mathbb{R}^{4}$. Given a ch-diagram $D$ of $\mathcal{L}$ with $V(D)=\left\{v_{1}, v_{2}, \ldots, v_{s}\right\}$, define a properly imbedded surface $F_{D}$ in $\mathbb{R}^{3} \times[-1,1] \subset \mathbb{R}^{4}$ 
by

$$
\left(\mathbb{R}_{t}^{3}, F_{D} \cap \mathbb{R}_{t}^{3}\right)= \begin{cases}\left(\mathbb{R}^{3}, L_{+}(D)\right), & \text { for } 0<t \leq 1 ; \\ \left(\mathbb{R}^{3}, L_{-}(D) \cup\left\{b_{1}, \ldots, b_{s}\right\}\right), & \text { for } t=0 ; \\ \left(\mathbb{R}^{3}, L_{-}(D)\right), & \text { for }-1 \leq t<0\end{cases}
$$

where $b_{i}(i=1, \ldots, s)$ is a band attached to $L_{-}(D)$ as shown in Figure2. Note that the links $L_{+}(D)$ and $L_{-}(D)$ are trivial links in $\mathbb{R}^{3}$, and thus we obtain a knotted surface $\overline{F_{D}}$ from $F_{D}$ by adding a set of 2-disks bounded by $L_{+}(D)$ in $\mathbb{R}_{1}^{3}$ and a set of 2 -disks bounded by $L_{-}(D)$ in $\mathbb{R}_{-1}^{3}$. Then the surface link $\overline{F_{D}}$ in $\mathbb{R}^{4}$ is equivalent to the surface $\mathcal{L}\left[8,14\right.$. Therefore, any surface link $\mathcal{L}$ in $\mathbb{R}^{4}$ can be represented by a ch-diagram $D$ and $\mathcal{L}$ can be completely reconstructed from its ch-diagram $D$ up to equivalence. Equivalent surface links in $\mathbb{R}^{4}$ may be represented by many different ch-diagrams.

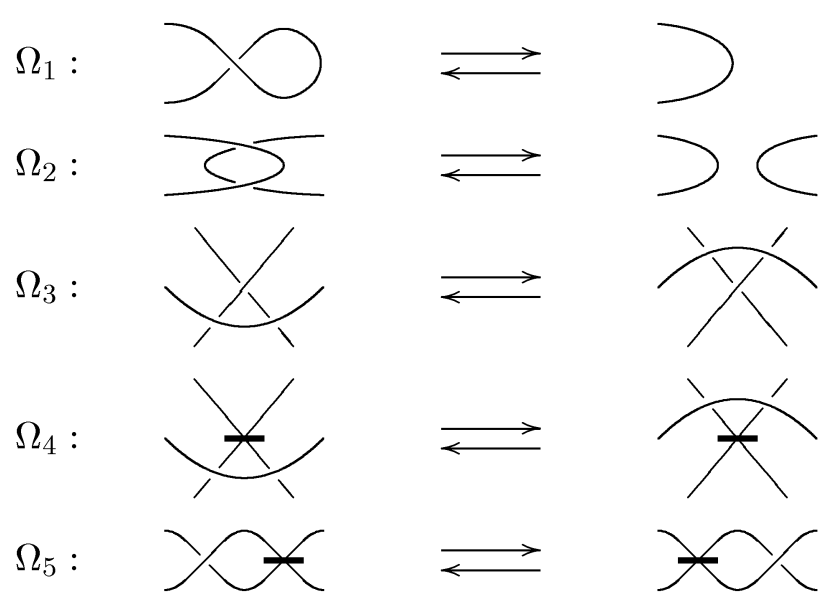

Figure 3. Moves of Type I

Definition 2.2. Two ch-diagrams $D$ and $D^{\prime}$ are said to be stably equivalent if they can be transformed into each other by a finite sequence of moves $\Omega_{i}(i=1,2, \ldots, 8)$ and $\Omega_{6}^{*}$ as shown in Figures 3 and 4 , and their mirror image moves.

Two surface links $\mathcal{L}$ and $\mathcal{L}^{\prime}$ in $\mathbb{R}^{4}$ are said to be stably equivalent if their chdiagrams are all stably equivalent.

The moves $\Omega_{1}, \Omega_{2}, \ldots, \Omega_{8}$ are local changes in a diagram, which were first introduced by Yoshikawa [27] in 1994. Note that the moves $\Omega_{1}, \Omega_{2}$ and $\Omega_{3}$ are just Reidemeister moves for classical knots and link diagrams and $\Omega_{6}^{*}$ is a mirror move of $\Omega_{6}$ with respect to the time direction, not in 3-space. It is known that all these moves and their mirror moves can be realized by ambient isotopies of $\mathbb{R}^{4}$ [14, 22, 26]. This implies that if two ch-diagrams are stably equivalent, then they represent equivalent surface links in $\mathbb{R}^{4}$.

Conjecture 2.3 (K. Yoshikawa, 27]). Two surface links $\mathcal{L}$ and $\mathcal{L}^{\prime}$ in $\mathbb{R}^{4}$ are equivalent if and only if they are stably equivalent, that is, their ch-diagrams are stably equivalent. 


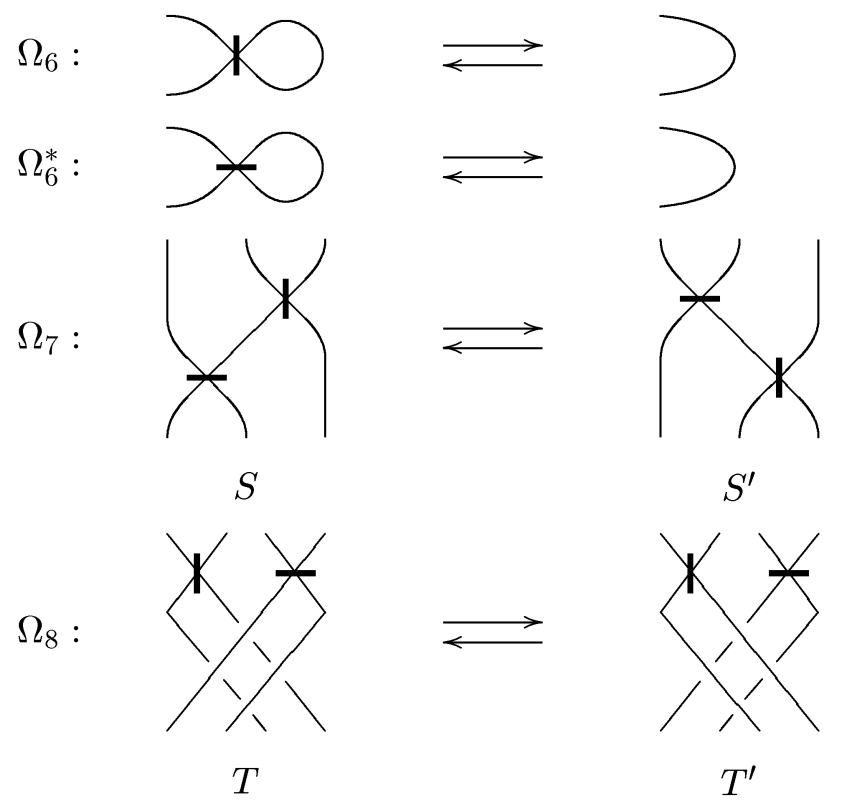

Figure 4. Moves of Type II

Remark 2.4. (1) In 2001, F. J. Swenton in his paper [26] claimed to have proved that Conjecture 2.3 above is true. In a private communication, S. Kamada told me that Swenton's paper has a gap and he has recovered it recently [12]. Consequently, any two ch-diagrams representing the same surface link are stably equivalent.

(2) In 27, Yoshikawa introduced the $c h$-index, denoted by $\operatorname{ch}(\mathcal{L})$, of a surface link $\mathcal{L}$, which is defined to be the minimum number $\operatorname{ch}(\mathcal{L})=\min _{D \in \mathcal{D}}(|V(D)|+|C(D)|)$, where $\mathcal{D}$ denotes the set of all ch-diagrams representing $\mathcal{L}$. Note that $\operatorname{ch}(\mathcal{L})$ is an ambient isotopy invariant of $\mathcal{L}$. Using this terminology, he made a table of 23 surface links in $\mathbb{R}^{4}$, denoted by $N_{k}^{g_{1}, g_{2}, \ldots, g_{n}}$, whose ch-indices are less than or equal to ten (see [27, Table I] or [15, Table F.7]), where $N_{k}^{g_{1}, g_{2}, \ldots, g_{n}}$ means the $k$-th surface with ch-index $N$ and $n$ components whose genera are $g_{1}, g_{2}, \ldots, g_{n}$. If $g_{i}<0$, then it means non-orientable genus. For a 2 -knot, $N_{k}^{0}$ is abbreviated by $N_{k}$.

\section{A POLYNOMIAL FOR A MARKED 4-VALENT SPATIAL GRAPH DIAGRAM}

Let $R$ be a commutative ring with the additive identity 0 and the multiplicative identity 1 and let $\hat{R}=R\left[A_{1}, \ldots, A_{m}\right], m \geq 0$, denote the ring of polynomials in the commuting variables $A_{1}, \ldots, A_{m}$ with coefficients in $R$. If $m=0$, then $\hat{R}=R$. Let [ ] be a regular or an ambient isotopy invariant of classical knots and links in 3 -space with the values in $\hat{R}$ and the following properties: for an element $\delta=\delta\left(A_{1}, \ldots, A_{m}\right) \in \hat{R}$ and an invertible element $\alpha=\alpha\left(A_{1}, \ldots, A_{m}\right) \in \hat{R}$,

$$
\text { [ } \left.\bigcirc]=\alpha[)],[\mathcal{O}]=\alpha^{-1}[)\right],[K \bigcirc]=\delta[K]
$$

where $K \bigcirc$ denotes any addition of a disjoint circle $\bigcirc$ to a classical knot or link diagram $K$. Notice that [ ] is an ambient isotopy invariant of classical knots and links if and only if $\alpha=1$. 
Definition 3.1. Let $D$ be a marked 4 -valent spatial graph diagram. Let $[[D]]=$ $[[D]]\left(A_{1}, \ldots, A_{m}, x, y, z, w\right)$ be a polynomial in $\hat{R}[x, y, z, w]$ defined by means of the two rules:

(L1) $[[D]]=[D]$ if $D$ is a classical knot or link diagram,

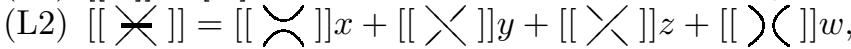

where $A_{1}, \ldots, A_{m}, x, y, z$ and $w$ are commuting variables and $\chi, \bigvee, X, X$, and $)$ ( are the small parts of five larger diagrams that are identical except at the five local sites indicated by the small parts.

Example 3.2. Let $0_{1}, 2_{1}^{-1}$ and $2_{1}^{1}$ be the trivial 2-knot, the positive standard projective plane and the standard torus of genus one in $\mathbb{R}^{4}$ and let $D_{1}, D_{2}$ and $D_{3}$ denote the ch-diagrams of $0_{1}, 2_{1}^{-1}$ and $2_{1}^{1}$ in Yoshikawa's table, respectively. Then

$$
\begin{aligned}
{\left[\left[D_{1}\right]\right]=[[\bigcirc]]=[\bigcirc], } \\
=[(つ)] x+[(>)] y+[(>)] z+[(2)] w \\
=\left(\alpha x+\delta z+\alpha^{-1} w\right)[\bigcirc]+y[
\end{aligned}
$$

and

$$
\begin{aligned}
& {\left[\left[D_{3}\right]\right]=[[\circlearrowleft]]=[[\precsim]] x}
\end{aligned}
$$

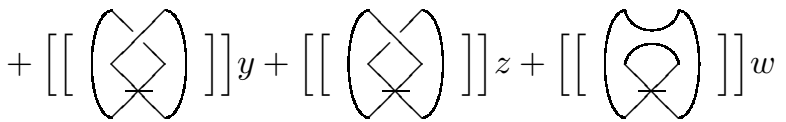

$$
\begin{aligned}
& =\left[(\mathcal{S}] x^{2}+[(\langle)] x y+[(\langle\}] x z+[\{\}] x w\right.
\end{aligned}
$$

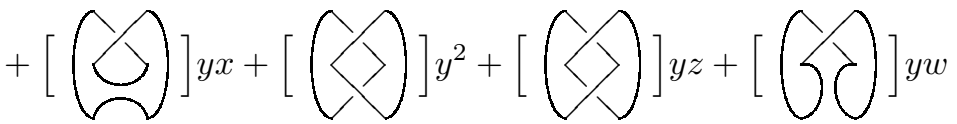

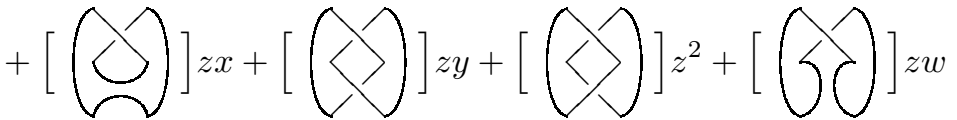

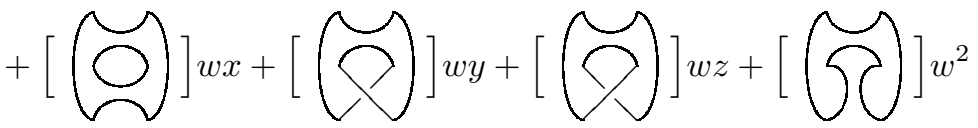

$$
\begin{aligned}
& =\left(x^{2}+2 \alpha^{-1} x y+2 \alpha x z+2 \alpha y w+2 \alpha^{-1} z w+w^{2}\right. \\
& \left.\left.\left.+\delta\left(y^{2}+z^{2}+2 x w\right)\right)[O]+y z([\bigotimes)]+[(\rangle)\right]\right) .
\end{aligned}
$$

Alternatively, we can define $[[D]]$ via a state-sum formula. A state $\sigma$ of $D$ is an assignment of $T_{\infty}, T_{-}, T_{+}$or $T_{0}$ to each marked vertex of $D$. Let $\mathcal{S}(D)$ denote 
the set of all states of $D$. For each state $\sigma \in \mathcal{S}(D)$, let $D_{\sigma}$ denote the classical knot or link diagram, called the state diagram, obtained from $D$ by replacing each

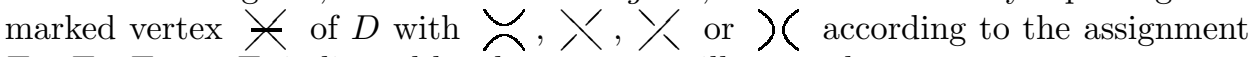
$T_{\infty}, T_{-}, T_{+}$, or $T_{0}$ indicated by the state $\sigma$ as illustrated:

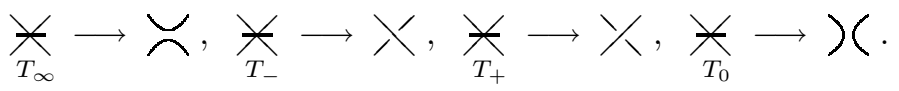

Since a marked vertex in the diagram $D$ is replaced in four ways, the set $\mathcal{S}(D)$ of all states of the diagram $D$ is in one-to-one correspondence with the disjoint

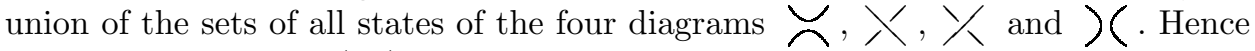
the defining relation (L2) gives at once the following state-sum formula for the polynomial $[[D]]$ :

$$
[[D]]\left(A_{1}, \ldots, A_{m}, x, y, z, w\right)=\sum_{\sigma \in \mathcal{S}(D)}\left[D_{\sigma}\right] x^{\sigma(\infty)} y^{\sigma(-)} z^{\sigma(+)} w^{\sigma(0)},
$$

where $\sigma(\infty), \sigma(-), \sigma(+)$ and $\sigma(0)$ denote the numbers of the assignment $T_{\infty}, T_{-}, T_{+}$ and $T_{0}$ of the state $\sigma$, respectively.

Lemma 3.3. Let $D$ be a marked 4-valent spatial graph diagram. Then for any regular (resp. ambient) isotopy invariant of classical knots and links, $[[D]]$ is invariant under $\Omega_{2}, \ldots, \Omega_{5}$ (resp. $\Omega_{1}, \Omega_{2}, \ldots, \Omega_{5}$ ) of the moves of Type I and their mirror moves.

Proof. Since [ ] is a regular (resp. an ambient) isotopy invariant of classical knots and links, it is obvious from the definition that $[[D]]$ is invariant under the classical Reidemeister moves $\Omega_{2}$ and $\Omega_{3}$ (resp. $\Omega_{1}, \Omega_{2}$ and $\Omega_{3}$ ). On the other hand, the figures 5 and 6 show the diagrammatic proof of the invariance of $[[D]]$ under the moves of $\Omega_{4}$ and $\Omega_{5}$, respectively. Similarly, one can easily see that $[[D]]$ is also invariant under the mirror moves of $\Omega_{2}, \ldots, \Omega_{5}$ (resp. $\left.\Omega_{1}, \Omega_{2}, \ldots, \Omega_{5}\right)$. This completes the proof.

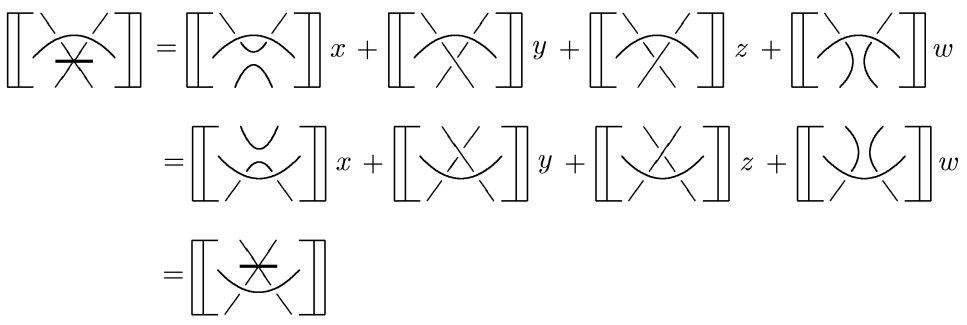

Figure 5. The move $\Omega_{4}$

The following Lemma 3.4 shows the behavior of $[[D]]$ under the moves $\Omega_{1}, \Omega_{6}$ and $\Omega_{6}^{*}$.

Lemma 3.4.

$$
\begin{aligned}
& {[[\bigcirc]]=\alpha[[\supset]],\left[[\bigcirc \bigcirc]=\alpha^{-1}[[\supset]],\right.} \\
& {[[\bigcirc]]=\left(\delta x+\alpha y+\alpha^{-1} z+w\right)[[\supset]],} \\
& {[[\mathcal{X}]]=\left(x+\alpha^{-1} y+\alpha z+\delta w\right)[[\supset]] .}
\end{aligned}
$$




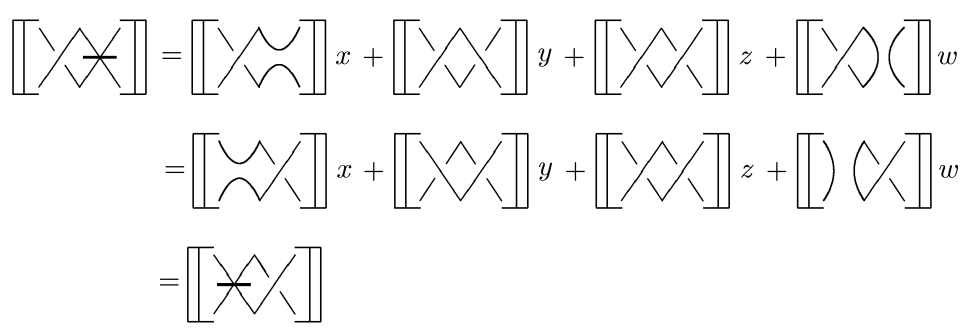

Figure 6 . The move $\Omega_{5}$

Proof. From the state-sum formula (3.2) together with (3.1), we see that

$$
\begin{aligned}
{[[\mathcal{\bigcirc}]] } & =\sum_{\sigma}\left[\mathcal{O}{ }_{\sigma}\right] x^{\sigma(\infty)} y^{\sigma(-)} z^{\sigma(+)} w^{\sigma(0)} \\
& =\sum_{\sigma \in \mathcal{S}())} \alpha\left[{ }_{\sigma}\right] x^{\sigma(\infty)} y^{\sigma(-)} z^{\sigma(+)} w^{\sigma(0)} \\
& =\alpha \sum_{\sigma \in \mathcal{S}())}\left[{ }_{\sigma}\right] x^{\sigma(\infty)} y^{\sigma(-)} z^{\sigma(+)} w^{\sigma(0)} \\
& =\alpha[[\supset]] .
\end{aligned}
$$

Similarly, [[ $\left.\lambda \bigcirc]=\alpha^{-1}[[]]\right]$. Now

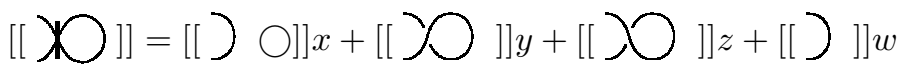

$$
\begin{aligned}
& =[[\mathcal{D}]]\left(\delta x+\alpha y+\alpha^{-1} z+w\right),
\end{aligned}
$$

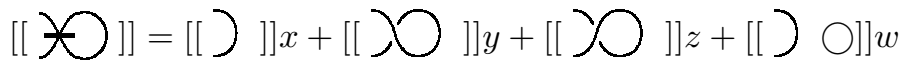

$$
\begin{aligned}
& =[[]]]\left(x+\alpha^{-1} y+\alpha z+\delta w\right) \text {. }
\end{aligned}
$$

This completes the proof.

We now investigate how the polynomial $[[D]]$ behaves under $\Omega_{7}$ and $\Omega_{8}$ of the moves of Type II. To do this we first introduce some notation. Let $\mathcal{T}_{n}$ denote the set of all $n$-tangle diagrams with or without marked vertices, let $\mathcal{T}_{n}^{c}$ denote the set of all classical $n$-tangle diagrams (without marked vertices), and let $B_{n}$ denote the geometric braid group on $n$-strings with geometric generators $\sigma_{1}, \sigma_{2}, \ldots, \sigma_{n-1}$. Then $B_{n} \subset \mathcal{T}_{n}^{c} \subset \mathcal{T}_{n}$. For two given tangles $x, y \in \mathcal{T}_{n}$, we denote by $x y$ the usual product of two tangles and by $x \circ y$ the knot or link that results from the $n$-tangles $x$ and $y$ by closing $n$ strings of $x$ and $y$ as shown in Figure 7, Let $e_{1}, e_{2}, \ldots, e_{n-1}$ denote the $n$-tangles shown in Figure 7 .

For our convenience we shall denote $f_{0}=1$, the trivial 3 -string braid, $f_{1}=$ $e_{1}, f_{2}=e_{2}, f_{3}=e_{1} e_{2}, f_{4}=e_{2} e_{1}$ in $\mathcal{T}_{3}^{c}$ and $g_{0}=1$, the trivial 4 -string braid, $g_{1}=e_{1}, g_{2}=e_{2}, g_{3}=e_{3}, g_{4}=e_{1} e_{2}, g_{5}=e_{1} e_{3}, g_{6}=e_{2} e_{1}, g_{7}=e_{2} e_{3}, g_{8}=e_{3} e_{2}, g_{9}=$ $e_{1} e_{2} e_{3}, g_{10}=e_{1} e_{3} e_{2}, g_{11}=e_{2} e_{1} e_{3}, g_{12}=e_{3} e_{2} e_{1}$, and $g_{13}=e_{2} e_{1} e_{3} e_{2}$ in $\mathcal{T}_{4}^{c}$.

Let $D$ be a marked 4 -valent spatial graph diagram. Suppose that $D^{\prime}$ is a diagram obtained from $D$ by a single move $\Omega_{7}$. By applying a finite number of the moves $\Omega_{i}(i=1,2, \ldots, 6), \Omega_{6}^{*}$, and their mirror moves if necessary, the diagram $D$ can be transformed to the diagram of the form $S \circ E$ as shown in Figure 8 , i.e., $D=S \circ E$, where $S$ is the 3 -tangle diagram in $\mathcal{T}_{3}$ with two marked vertices, say $v_{1}, v_{2}$ (the 


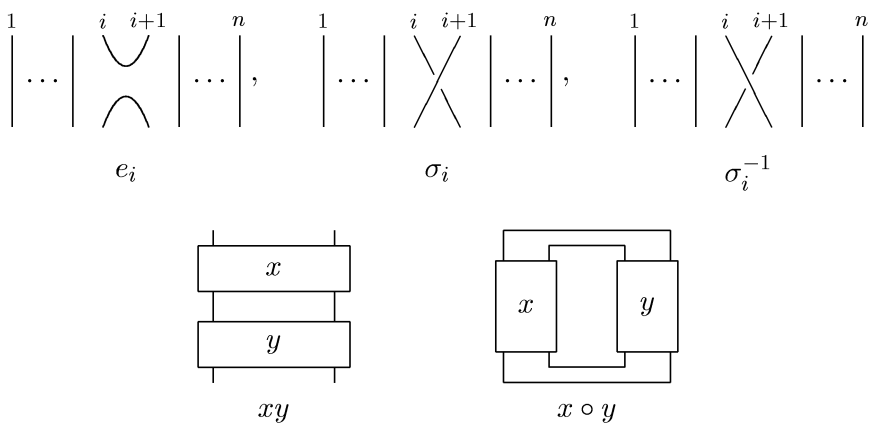

Figure 7

left-hand side of the move $\Omega_{7}$ in Figure (4), and $E$ is a 3 -tangle diagram in $\mathcal{T}_{3}$ with marked vertices equivalent to $D-S$ under the moves $\Omega_{i}(i=1,2, \ldots, 6)$. We also assume that $D^{\prime}=S^{\prime} \circ E$ as shown in Figure 8, where $S^{\prime}$ is the 3-tangle diagram in $\mathcal{T}_{3}$ with two marked vertices, say $v_{1}^{\prime}, v_{2}^{\prime}$ (the right-hand side of the move $\Omega_{7}$ in Figure 4).
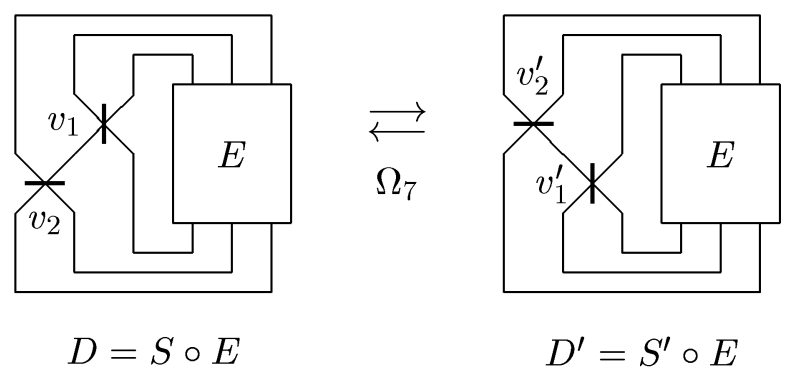

FigURE 8

Let $\left\{\tau_{k} \mid k=1,2, \ldots, 16\right\}$ be the set of all states of $S$ and let $\left\{\tau_{k}^{\prime} \mid k=1,2, \ldots, 16\right\}$ be the set of all states of $S^{\prime}$ given by $\tau_{k}^{\prime}\left(v_{i}^{\prime}\right)=\tau_{k}\left(v_{i}\right)$ for each $i=1,2$. For each $\tau_{k}$ and $\tau_{k}^{\prime}$, let $S_{\tau_{k}} \circ E$ and $S_{\tau_{k}^{\prime}}^{\prime} \circ E$ be the diagrams obtained from $S \circ E$ and $S^{\prime} \circ E$,

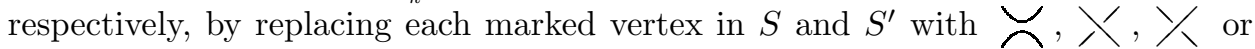
) ( according to $\tau_{k}$ and $\tau_{k}^{\prime}$. Table 1 lists all these diagrams and corresponding monomials, where $M\left(\tau_{k}\right)=x^{\tau_{k}(\infty)} y^{\tau_{k}(-)} z^{\tau_{k}(+)} w^{\tau_{k}(0)}=M\left(\tau_{k}^{\prime}\right)$. Define

$$
\begin{aligned}
& \Delta_{7}\left(A_{1}, \ldots, A_{m}, x, y, z, w ; U\right)=x w\left(\left[f_{4} \circ U\right]-\left[f_{3} \circ U\right]\right) \\
& +z w\left(\left[f_{2} \sigma_{1} \circ U\right]-\left[\sigma_{1} f_{2} \circ U\right]\right)+y w\left(\left[f_{2} \sigma_{1}^{-1} \circ U\right]-\left[\sigma_{1}^{-1} f_{2} \circ U\right]\right) \\
& +x y\left(\left[\sigma_{2} f_{1} \circ U\right]-\left[f_{1} \sigma_{2} \circ U\right]\right)+x z\left(\left[\sigma_{2}^{-1} f_{1} \circ U\right]-\left[f_{1} \sigma_{2}^{-1} \circ U\right]\right) \\
& +y^{2}\left(\left[\sigma_{2} \sigma_{1}^{-1} \circ U\right]-\left[\sigma_{1}^{-1} \sigma_{2} \circ U\right]\right)+z^{2}\left(\left[\sigma_{2}^{-1} \sigma_{1} \circ U\right]-\left[\sigma_{1} \sigma_{2}^{-1} \circ U\right]\right) \\
& +y z\left(\left[\sigma_{2} \sigma_{1} \circ U\right]-\left[\sigma_{1} \sigma_{2} \circ U\right]+\left[\sigma_{2}^{-1} \sigma_{1}^{-1} \circ U\right]-\left[\sigma_{1}^{-1} \sigma_{2}^{-1} \circ U\right]\right) .
\end{aligned}
$$

In what follows we denote by $\mathbb{E}$ an extension ring of the $\operatorname{ring} R\left[A_{1}, \ldots, A_{m}\right.$, $x, y, z, w]$, or simply $\hat{R}[x, y, z, w]$, of polynomials in commuting variables $A_{1}, \ldots, A_{m}$, $x, y, z, w$ with coefficients in $R$, otherwise specified. 
TABLE 1

\begin{tabular}{|l|ll|l|l|l|}
\hline$k$ & $\tau_{k}\left(v_{1}\right)$ & $\tau_{k}\left(v_{2}\right)$ & $S_{\tau_{k}} \circ E$ & $S_{\tau_{k}^{\prime}}^{\prime} \circ E$ & $M\left(\tau_{k}\right)$ \\
\hline 1 & $T_{\infty}$ & $T_{\infty}$ & $f_{1} \circ E$ & $f_{1} \circ E$ & $x^{2}$ \\
2 & $T_{\infty}$ & $T_{-}$ & $\sigma_{1}^{-1} \circ E$ & $\sigma_{1}^{-1} \circ E$ & $x y$ \\
3 & $T_{\infty}$ & $T_{+}$ & $\sigma_{1} \circ E$ & $\sigma_{1} \circ E$ & $x z$ \\
4 & $T_{\infty}$ & $T_{0}$ & $f_{0} \circ E$ & $f_{0} \circ E$ & $x w$ \\
5 & $T_{-}$ & $T_{\infty}$ & $\sigma_{2} f_{1} \circ E$ & $f_{1} \sigma_{2} \circ E$ & $y x$ \\
6 & $T_{-}$ & $T_{-}$ & $\sigma_{2} \sigma_{1}^{-1} \circ E$ & $\sigma_{1}^{-1} \sigma_{2} \circ E$ & $y^{2}$ \\
7 & $T_{-}$ & $T_{+}$ & $\sigma_{2} \sigma_{1} \circ E$ & $\sigma_{1} \sigma_{2} \circ E$ & $y z$ \\
8 & $T_{-}$ & $T_{0}$ & $\sigma_{2} \circ E$ & $\sigma_{2} \circ E$ & $y w$ \\
9 & $T_{+}$ & $T_{\infty}$ & $\sigma_{2}^{-1} f_{1} \circ E$ & $f_{1} \sigma_{2}^{-1} \circ E$ & $z x$ \\
10 & $T_{+}$ & $T_{-}$ & $\sigma_{2}^{-1} \sigma_{1}^{-1} \circ E$ & $\sigma_{1}^{-1} \sigma_{2}^{-1} \circ E$ & $z y$ \\
11 & $T_{+}$ & $T_{+}$ & $\sigma_{2}^{-1} \sigma_{1} \circ E$ & $\sigma_{1} \sigma_{2}^{-1} \circ E$ & $z^{2}$ \\
12 & $T_{+}$ & $T_{0}$ & $\sigma_{2}^{-1} \circ E$ & $\sigma_{2}^{-1} \circ E$ & $z w$ \\
13 & $T_{0}$ & $T_{\infty}$ & $f_{4} \circ E$ & $f_{3} \circ E$ & $w x$ \\
14 & $T_{0}$ & $T_{-}$ & $f_{2} \sigma_{1}^{-1} \circ E$ & $\sigma_{1}^{-1} f_{2} \circ E$ & $w y$ \\
15 & $T_{0}$ & $T_{+}$ & $f_{2} \sigma_{1} \circ E$ & $\sigma_{1} f_{2} \circ E$ & $w z$ \\
16 & $T_{0}$ & $T_{0}$ & $f_{2} \circ E$ & $f_{2} \circ E$ & $w^{2}$ \\
\hline
\end{tabular}

Lemma 3.5. Let $D$ be a marked 4-valent spatial graph diagram. Suppose that $(\mathbf{a}, \mathbf{s})=\left(a_{1}, \ldots, a_{m}, s_{1}, s_{2}, s_{3}, s_{4}\right) \in \mathbb{E}^{m+4}$ such that $\Delta_{7}\left(a_{1}, \ldots, a_{m}, s_{1}, s_{2}, s_{3}, s_{4} ; U\right)$ $=0$ for any classical 3 -tangle $U \in \mathcal{T}_{3}^{c}$. Then $[[D]](\mathbf{a}, \mathbf{s})=[[D]]\left(a_{1}, \ldots, a_{m}, s_{1}, s_{2}, s_{3}, s_{4}\right)$ is an invariant of the move $\Omega_{7}$.

Proof. Let $\mathcal{S}(E)$ be the set of all states of the 3-tangle diagram $E$ in the diagram $D$ in Figure 8, Then it follows that

$$
\begin{aligned}
{[[D]] } & =\sum_{k=1}^{16} M\left(\tau_{k}\right)\left[\left[S_{\tau_{k}} \circ E\right]\right] \\
& =\sum_{k=1}^{16} M\left(\tau_{k}\right)\left(\sum_{\sigma \in \mathcal{S}(E)}\left[S_{\tau_{k}} \circ E_{\sigma}\right] x^{\sigma(\infty)} y^{\sigma(-)} z^{\sigma(+)} w^{\sigma(0)}\right) \\
& =\sum_{\sigma \in \mathcal{S}(E)}\left(\sum_{k=1}^{16} M\left(\tau_{k}\right)\left[S_{\tau_{k}} \circ E_{\sigma}\right]\right) x^{\sigma(\infty)} y^{\sigma(-)} z^{\sigma(+)} w^{\sigma(0)}, \\
{\left[\left[D^{\prime}\right]\right] } & =\sum_{k=1}^{16} M\left(\tau_{k}^{\prime}\right)\left[\left[S_{\tau_{k}^{\prime}}^{\prime} \circ E\right]\right] \\
& =\sum_{k=1}^{16} M\left(\tau_{k}\right)\left(\sum_{\sigma \in \mathcal{S}(E)}\left[S_{\tau_{k}^{\prime}}^{\prime} \circ E_{\sigma}\right] x^{\sigma(\infty)} y^{\sigma(-)} z^{\sigma(+)} w^{\sigma(0)}\right) \\
& =\sum_{\sigma \in \mathcal{S}(E)}\left(\sum_{k=1}^{16} M\left(\tau_{k}\right)\left[S_{\tau_{k}^{\prime}}^{\prime} \circ E_{\sigma}\right]\right) x^{\sigma(\infty)} y^{\sigma(-)} z^{\sigma(+)} w^{\sigma(0)} .
\end{aligned}
$$


From Table 1, we obtain that for each $\sigma \in \mathcal{S}(E)$,

$$
\sum_{k=1}^{16} M\left(\tau_{k}\right)\left[S_{\tau_{k}} \circ E_{\sigma}\right]-\sum_{k=1}^{16} M\left(\tau_{k}\right)\left[S_{\tau_{k}^{\prime}}^{\prime} \circ E_{\sigma}\right]=\Delta_{7}\left(A_{1}, \ldots, A_{m}, x, y, z, w ; E_{\sigma}\right) .
$$

Hence it follows that

$$
[[D]]-\left[\left[D^{\prime}\right]\right]=\sum_{\sigma \in \mathcal{S}(E)} \Delta_{7}\left(A_{1}, \ldots, A_{m}, x, y, z, w ; E_{\sigma}\right) x^{\sigma(\infty)} y^{\sigma(-)} z^{\sigma(+)} w^{\sigma(0)} .
$$

Since $\Delta_{7}\left(a_{1}, \ldots, a_{m}, s_{1}, s_{2}, s_{3}, s_{4} ; U\right)=0$ for any classical 3 -tangle $U \in \mathcal{T}_{3}^{c}$, we have $\Delta_{7}\left(a_{1}, \ldots, a_{m}, s_{1}, s_{2}, s_{3}, s_{4} ; E_{\sigma}\right)=0$ for each $\sigma \in \mathcal{S}(E)$ and thus $[[D]](\mathbf{a}, \mathbf{s})=$ $\left[\left[D^{\prime}\right]\right](\mathbf{a}, \mathbf{s})$. This completes the proof.

Next, suppose that $D^{\prime}$ is a diagram obtained from a marked 4-valent spatial graph diagram $D$ by a single move $\Omega_{8}$. By applying a finite number of the moves $\Omega_{i}(i=1,2, \ldots, 6), \Omega_{6}^{*}$, and their mirror moves if necessary, the diagram $D$ can be transformed to the diagram of the form $T \circ F$ as shown in Figure 9, i.e., $D=T \circ F$, where $T$ is the 4 -tangle diagram in $\mathcal{T}_{4}$ with two marked vertices, say $v_{1}, v_{2}$ (the left-hand side of the move $\Omega_{8}$ in Figure 4 ), and $F$ is a 4 -tangle diagram in $\mathcal{T}_{4}$ with marked vertices equivalent to $D-T$ under the moves $\Omega_{i}(i=1,2, \ldots, 6)$ or their mirror moves. We also assume that $D^{\prime}=T^{\prime} \circ F$ as shown in Figure 9, where $T^{\prime}$ is the 4 -tangle diagram in $\mathcal{T}_{4}$ with two marked vertices, say $v_{1}^{\prime}, v_{2}^{\prime}$ (the right-hand side of the move $\Omega_{8}$ in Figure (4).

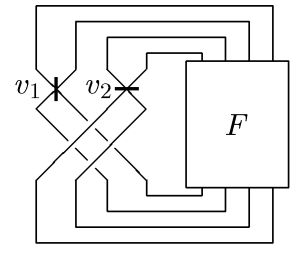

$D=T \circ F$

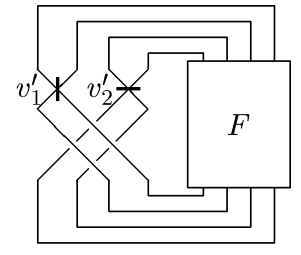

$D^{\prime}=T^{\prime} \circ F$

Figure 9

Let $\left\{\tau_{k} \mid k=1,2, \ldots, 16\right\}$ be the set of all states of $T$ and let $\left\{\tau_{k}^{\prime} \mid k=1,2, \ldots, 16\right\}$ be the set of all states of $T^{\prime}$ given by $\tau_{k}^{\prime}\left(v_{i}^{\prime}\right)=\tau_{k}\left(v_{i}\right)$ for each $i=1,2$. For each $\tau_{k}$ and $\tau_{k}^{\prime}$, let $T_{\tau_{k}} \circ F$ and $T_{\tau_{k}^{\prime}}^{\prime} \circ F$ be the diagrams obtained from $T \circ F$ and $T^{\prime} \circ F$,

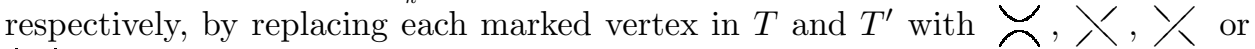
) ( according to $\tau_{k}$ and $\tau_{k}^{\prime}$. Table 2 lists all these diagrams and corresponding monomials, where $\beta=\sigma_{2} \sigma_{1} \sigma_{3} \sigma_{2}$ and $\beta^{*}=\sigma_{2}^{-1} \sigma_{1}^{-1} \sigma_{3}^{-1} \sigma_{2}^{-1}$ are 4-braids. Define

$$
\begin{aligned}
& \Delta_{8}\left(A_{1}, \ldots, A_{m}, x, y, z, w ; V\right)=x w\left([\beta \circ V]-\left[\beta^{*} \circ V\right]\right) \\
& +y w\left(\left[\sigma_{1} \beta \circ V\right]-\left[\sigma_{1} \beta^{*} \circ V\right]\right)+z w\left(\left[\sigma_{1}^{-1} \beta \circ V\right]-\left[\sigma_{1}^{-1} \beta^{*} \circ V\right]\right) \\
& \left.+y z\left(\left[\sigma_{1} \sigma_{3} \beta \circ V\right]-\left[\sigma_{1} \sigma_{3} \beta^{*} \circ V\right]\right)+\left[\sigma_{1}^{-1} \sigma_{3}^{-1} \beta \circ V\right]-\left[\sigma_{1}^{-1} \sigma_{3}^{-1} \beta^{*} \circ V\right]\right) \\
& +y^{2}\left(\left[\sigma_{1} \sigma_{3}^{-1} \beta \circ V\right]-\left[\sigma_{1} \sigma_{3}^{-1} \beta^{*} \circ V\right]\right)+z^{2}\left(\left[\sigma_{1}^{-1} \sigma_{3} \beta \circ V\right]-\left[\sigma_{1}^{-1} \sigma_{3} \beta^{*} \circ V\right]\right) \\
& +x z\left(\left[\sigma_{3} \beta \circ V\right]-\left[\sigma_{3} \beta^{*} \circ V\right]\right)+x y\left(\left[\sigma_{3}^{-1} \beta \circ V\right]-\left[\sigma_{3}^{-1} \beta^{*} \circ V\right]\right) .
\end{aligned}
$$


TABLE 2

\begin{tabular}{|l|ll|l|l|l|}
\hline$k$ & $\tau_{k}\left(v_{1}\right)$ & $\tau_{k}\left(v_{2}\right)$ & $T_{\tau_{k}} \circ F$ & $T_{\tau_{k}^{\prime}}^{\prime} \circ F$ & $M\left(\tau_{k}\right)$ \\
\hline 1 & $T_{\infty}$ & $T_{\infty}$ & $g_{12} \circ F$ & $g_{12} \circ F$ & $x^{2}$ \\
2 & $T_{\infty}$ & $T_{-}$ & $\sigma_{3}^{-1} \beta \circ F$ & $\sigma_{3}^{-1} \beta^{*} \circ F$ & $x y$ \\
3 & $T_{\infty}$ & $T_{+}$ & $\sigma_{3} \beta \circ F$ & $\sigma_{3} \beta^{*} \circ F$ & $x z$ \\
4 & $T_{\infty}$ & $T_{0}$ & $\beta \circ F$ & $\beta^{*} \circ F$ & $x w$ \\
5 & $T_{-}$ & $T_{\infty}$ & $\sigma_{1} g_{12} \circ F$ & $\sigma_{1} g_{12} \circ F$ & $y x$ \\
6 & $T_{-}$ & $T_{-}$ & $\sigma_{1} \sigma_{3}^{-1} \beta \circ F$ & $\sigma_{1} \sigma_{3}^{-1} \beta^{*} \circ F$ & $y^{2}$ \\
7 & $T_{-}$ & $T_{+}$ & $\sigma_{1} \sigma_{3} \beta \circ F$ & $\sigma_{1} \sigma_{3} \beta^{*} \circ F$ & $y z$ \\
8 & $T_{-}$ & $T_{0}$ & $\sigma_{1} \beta \circ F$ & $\sigma_{1} \beta^{*} \circ F$ & $y w$ \\
9 & $T_{+}$ & $T_{\infty}$ & $\sigma_{1}^{-1} g_{12} \circ F$ & $\sigma_{1}^{-1} g_{12} \circ F$ & $z x$ \\
10 & $T_{+}$ & $T_{-}$ & $\sigma_{1}^{-1} \sigma_{3}^{-1} \beta \circ F$ & $\sigma_{1}^{-1} \sigma_{3}^{-1} \beta^{*} \circ F$ & $z y$ \\
11 & $T_{+}$ & $T_{+}$ & $\sigma_{1}^{-1} \sigma_{3} \beta \circ F$ & $\sigma_{1}^{-1} \sigma_{3} \beta^{*} \circ F$ & $z^{2}$ \\
12 & $T_{+}$ & $T_{0}$ & $\sigma_{1}^{-1} \beta \circ F$ & $\sigma_{1}^{-1} \beta^{*} \circ F$ & $z w$ \\
13 & $T_{0}$ & $T_{\infty}$ & $g_{5} \circ F$ & $g_{5} \circ F$ & $w x$ \\
14 & $T_{0}$ & $T_{-}$ & $\sigma_{3}^{-1} g_{9} \circ F$ & $\sigma_{3}^{-1} g_{9} \circ F$ & $w y$ \\
15 & $T_{0}$ & $T_{+}$ & $\sigma_{3} g_{9} \circ F$ & $\sigma_{3} g_{9} \circ F$ & $w z$ \\
16 & $T_{0}$ & $T_{0}$ & $g_{9} \circ F$ & $g_{9} \circ F$ & $w^{2}$ \\
\hline
\end{tabular}

Lemma 3.6. Let $D$ be a marked 4-valent spatial graph diagram. Suppose that $(\mathbf{a}, \mathbf{s})=\left(a_{1}, \ldots, a_{m}, s_{1}, s_{2}, s_{3}, s_{4}\right) \in \mathbb{E}^{m+4}$ such that $\Delta_{8}\left(a_{1}, \ldots, a_{m}, s_{1}, s_{2}, s_{3}, s_{4} ; V\right)$ $=0$ for any classical 4 -tangle $V \in \mathcal{T}_{4}^{c}$. Then $[[D]](\mathbf{a}, \mathbf{s})=[[D]]\left(a_{1}, \ldots, a_{m}, s_{1}, s_{2}, s_{3}, s_{4}\right)$ is an invariant of the move $\Omega_{8}$.

Proof. By the proof of Lemma 3.5 with slight modifications, it is easily seen that $[[D]](\mathbf{a}, \mathbf{s})$ is invariant under the move $\Omega_{8}$.

Theorem 3.7. Let $D$ be a marked 4-valent spatial graph diagram, let [] be a regular (resp. an ambient) isotopy invariant of classical links, and let $\mathbf{V}(\Delta ;[])$ be the set of all $(m+4)$-tuples $(\mathbf{a}, \mathbf{s})=\left(a_{1}, \ldots, a_{m}, s_{1}, s_{2}, s_{3}, s_{4}\right) \in \mathbb{E}^{m+4}$ satisfying the system:

$$
(\Delta ;[])=\left\{\begin{array}{l}
\Delta_{7}\left(A_{1}, \ldots, A_{m}, x, y, z, w ; U\right)=0 \quad \text { for all } U \in \mathcal{T}_{3}^{c} ; \\
\Delta_{8}\left(A_{1}, \ldots, A_{m}, x, y, z, w ; V\right)=0 \quad \text { for all } V \in \mathcal{T}_{4}^{c} .
\end{array}\right.
$$

Then for any $(\mathbf{a}, \mathbf{s}) \in \mathbf{V}(\Delta ;[]),[[D]](\mathbf{a}, \mathbf{s})$ is an invariant of all Yoshikawa moves and their mirror moves, except the moves $\Omega_{1}, \Omega_{6}$ and $\Omega_{6}^{*}$ (resp. the moves $\Omega_{6}$ and $\left.\Omega_{6}^{*}\right)$.

Proof. From Lemmas 3.3, 3.5 and 3.6, the result follows at once.

It is worth noting that if the ring $R$ has the algebraically closed extension field $\mathbb{F}$, then, by the Hilbert Basis Theorem [4], $\mathbf{V}(\Delta ;[])$ is completely determined by a finite number of polynomials, say $p_{1}, p_{2}, \ldots, p_{r}$, in $\mathbb{F}\left[A_{1}, \ldots, A_{m}, x, y, z, w\right]$, that is,

$$
\mathbf{V}(\Delta ;[])=\left\{(\mathbf{a}, \mathbf{s}) \in \mathbb{F}^{m+4} \mid p_{i}(\mathbf{a}, \mathbf{s})=0, i=1,2, \ldots, r\right\} .
$$




\section{INVARIANTS OF SURFACE LINKS VIA CLASSICAL LINK INVARIANTS}

In this section, we shall normalize the value $[[D]](\mathbf{a}, \mathbf{s})$ of Theorem 3.7 so that it is also invariant under the moves $\Omega_{1}, \Omega_{6}$ and $\Omega_{6}^{*}$. From now on, let $D$ be a ch-diagram and let $L_{-}(D)$ and $L_{+}(D)$ be the classical link diagrams defined as in Figure 2 . Suppose that

$$
L_{-}(D)=U_{1}^{-} \cup \cdots \cup U_{m}^{-} \text {and } L_{+}(D)=U_{1}^{+} \cup \cdots \cup U_{n}^{+} .
$$

Recall that $L_{-}(D)$ and $L_{+}(D)$ are trivial link diagrams and so all $U_{i}^{-}(1 \leq i \leq m)$ and $U_{j}^{+}(1 \leq j \leq n)$ are unknotted. Let

$$
\begin{aligned}
& t_{-}(D)=w\left(L_{-}(D)\right)=\sum_{i=1}^{m} w\left(U_{i}^{-}\right), \\
& t_{+}(D)=w\left(L_{+}(D)\right)=\sum_{j=1}^{n} w\left(U_{j}^{+}\right),
\end{aligned}
$$

where $w\left(U_{i}^{-}\right)$and $w\left(U_{j}^{+}\right)$denote the usual writhes of the components $U_{i}^{-}$and $U_{j}^{+}$ of $L_{-}(D)$ and $L_{+}(D)$ with arbitrary chosen orientations, respectively. Note that for a knot diagram, the writhe is independent of the choice of orientation. We then define $t(D)$ to be an integer given by the formula

$$
t(D)=t_{+}(D)+t_{-}(D) .
$$

Next, $\mu_{+}(D)$ and $\mu_{-}(D)$ denote the numbers of components of the links $L_{+}(D)$ and $L_{-}(D)$, respectively. Then we define $e(D)$ to be an integer given by the formula

$$
e(D)=\mu_{+}(D)-\mu_{-}(D)
$$

Lemma 4.1. Let $D$ be any ch-diagram. Then

(1) $t_{+}(D), t_{-}(D)$ and $t(D)$ are invariant under all moves $\Omega_{2}, \ldots, \Omega_{8}, \Omega_{6}^{*}$. For the move $\Omega_{1}$,

$$
\begin{aligned}
& t_{+}(\bigcirc)=t_{+}()+1, \quad t_{+}(\bigcirc)=t_{+}(\supset)-1, \\
& t_{-}(\bigcirc)=t_{-}()+1, \quad t_{-}(\bigcirc)=t_{-}(\supset)-1 \text {, } \\
& t(0)=t(0)+2, t(\bigcirc)=t()-2 .
\end{aligned}
$$

(2) $e(D)$ is invariant under all moves $\Omega_{1}, \ldots, \Omega_{5}, \Omega_{7}, \Omega_{8}$. For the moves $\Omega_{6}$ and $\Omega_{6}^{*}$

$$
e(X)=e(\supset)+1, e(夫))=e(D)-1
$$

Proof. (1) Since the writhe is a regular isotopy invariant for classical link diagrams, it is immediate from the definition that $t_{+}(D)$ and $t_{-}(D)$ are invariant under the moves $\Omega_{2}$ and $\Omega_{3}$ and so is $t(D)$. The following Figure 10 shows the diagrammatic proof of the invariance of both $t_{+}(D)$ and $t_{-}(D)$ under the moves $\Omega_{4}, \ldots, \Omega_{8}, \Omega_{6}^{*}$, which implies the invariance of $t(D)$ under the moves. In Figure 10, each equality 
results from the obvious fact that before and after the move under consideration, the writhes of the corresponding links $L_{-}(D)$ and $L_{+}(D)$ are not changed. By a similar argument, we see that $t_{+}(D), t_{-}(D)$ and $t(D)$ are invariant under the mirror moves.

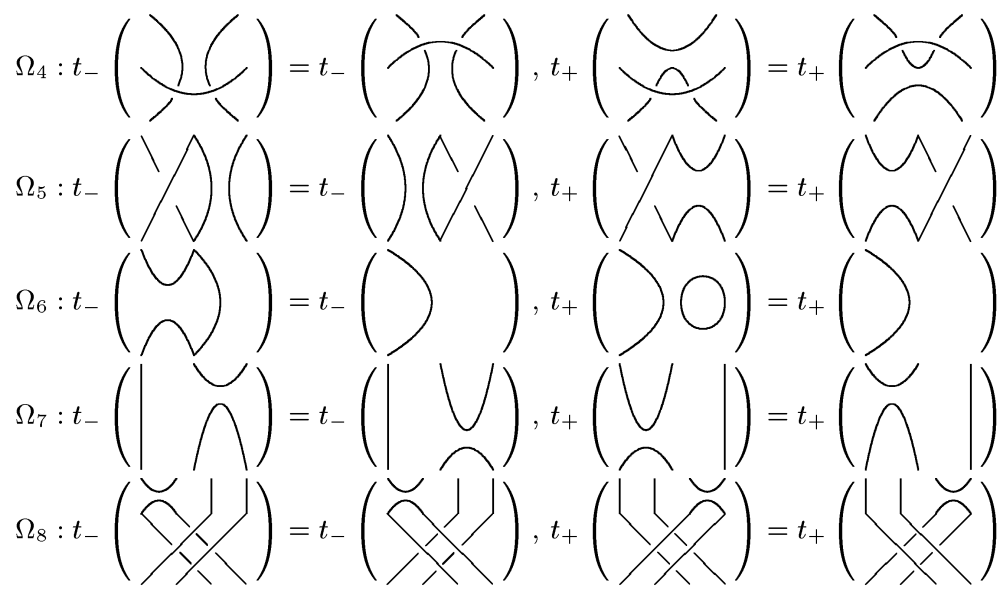

FIGURE 10

For the move $\Omega_{1}$, (4.1) and (4.2) are straightforward from the definition. Now for (4.3),

$$
\begin{aligned}
t(\mathcal{O}) & =t_{+}(\mathcal{O})+t_{-}(\mathcal{O}) \\
& =t_{+}(\supset)+1+t_{-}(\supset)+1 \\
& =t(\supset)+2 .
\end{aligned}
$$

Similarly, $t(\lambda \bigcirc)=t()-2$.

(2) Since the number of components of a classical link is an ambient isotopy invariant, it is obvious that both $\mu_{+}(D)$ and $\mu_{-}(D)$ are invariant under the moves $\Omega_{1}, \Omega_{2}$ and $\Omega_{3}$ and so is $e(D)$. From Figure 10 again, it is easy to check that $e(D)$ is not changed by all moves $\Omega_{i}$ and their mirror moves, except the moves $\Omega_{6}$ and $\Omega_{6}^{*}$. For the proof of the identity (4.4) for the moves $\Omega_{6}$ and $\Omega_{6}^{*}$, we first observe that

$$
\begin{aligned}
& L_{+}(X) \sim L_{+}(\supset) \cup \bigcirc, L_{-}(X) \sim L_{-}(\supset), \\
& L_{+}(\not) \sim L_{+}(\supset), L_{-}(\not \bigcirc) \sim L_{-}(\supset) \cup \bigcirc,
\end{aligned}
$$


where $\sim$ means ambient isotopic. From this obvious observation, we have

$$
\begin{aligned}
e(X) & =\mu_{+}(\nsupseteq \bigcirc)-\mu_{-}(X) \\
& =\left(\mu_{+}(\supset)+1\right)-\mu_{-}(\supset) \\
& =e(\supset)+1
\end{aligned}
$$

and

$$
\begin{aligned}
& e(大)=\mu_{+}(\text {关 })-\mu_{-}(\text {无) } \\
& =\mu_{+}(\supset)-\left(\mu_{-}(\supset)+1\right) \\
& =e())-1 \text {. }
\end{aligned}
$$

This completes the proof of Lemma 4.1

Lemma 4.2. Let $D$ be any ch-diagram and let [] be a regular or an ambient isotopy invariant of classical links. Then for any $(\mathbf{a}, \mathbf{s}) \in \mathbf{V}(\Delta ;[])$ such that $\alpha(\mathbf{a})$ is invertible,

$$
J_{D}(\mathbf{a}, \mathbf{s})=\alpha(\mathbf{a})^{-t_{+}(D)}[[D]](\mathbf{a}, \mathbf{s})
$$

is an invariant of all Yoshikawa moves $\Omega_{1}, \ldots, \Omega_{5}, \Omega_{7}, \Omega_{8}$ and their mirror moves, except the moves $\Omega_{6}$ and $\Omega_{6}^{*}$.

Proof. By Theorem 3.7 and Lemma 4.1 (1), it is immediate that $J_{D}(\mathbf{a}, \mathbf{s})$ is invariant under the moves $\Omega_{2}, \Omega_{3}, \Omega_{4}, \Omega_{5}, \Omega_{7}, \Omega_{8}$ and their mirror moves. From Lemma 3.4 together with (4.1), it follows that

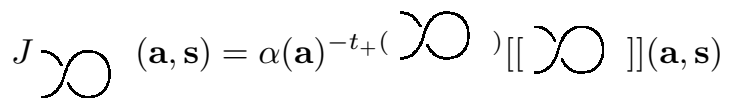

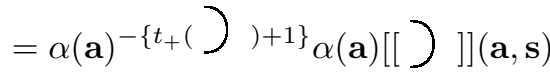

$$
\begin{aligned}
& =\alpha(\mathbf{a})^{\left.-t_{+}()^{\prime}\right)}[[]](\mathbf{a}, \mathbf{s}) \\
& =J(\mathbf{a}, \mathbf{s})
\end{aligned}
$$

and

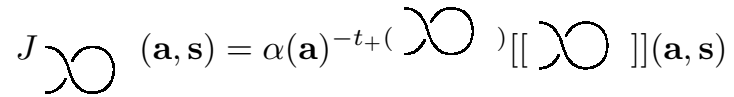

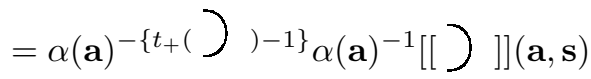

$$
\begin{aligned}
& \left.=\alpha(\mathbf{a})^{-t_{+}()}[[]]\right](\mathbf{a}, \mathbf{s}) \\
& =J(\mathbf{a}, \mathbf{s}) \text {. }
\end{aligned}
$$

This proves the invariance of $J_{D}(\mathbf{a}, \mathbf{s})$ under the move $\Omega_{1}$ and thus completes the proof. 
Now we are ready to state the main theorems of this section. For $(\mathbf{a}, \mathbf{s})=$ $\left(a_{1}, \ldots, a_{m}, s_{1}, s_{2}, s_{3}, s_{4}\right) \in \mathbf{V}(\Delta ;[])$, define

$$
\begin{aligned}
& \lambda_{1}(\mathbf{a}, \mathbf{s})=\delta(\mathbf{a}) s_{1}+\alpha(\mathbf{a}) s_{2}+\alpha(\mathbf{a})^{-1} s_{3}+s_{4}, \\
& \lambda_{2}(\mathbf{a}, \mathbf{s})=s_{1}+\alpha(\mathbf{a})^{-1} s_{2}+\alpha(\mathbf{a}) s_{3}+\delta(\mathbf{a}) s_{4},
\end{aligned}
$$

and

$$
\langle\langle D\rangle\rangle(\mathbf{a}, \mathbf{s})=[[D]](\mathbf{a}, \mathbf{s})\left[\left[D^{*}\right]\right](\mathbf{a}, \mathbf{s}),
$$

where $D^{*}$ is the ch-diagram obtained from a ch-diagram $D$ with respect to the opposite time direction, that is, by replacing all vertex markers in $D$ as shown:

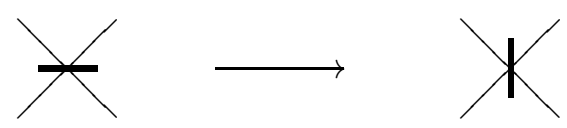

Theorem 4.3. Let $D$ be any ch-diagram of a surface link $\mathcal{L}$ in $\mathbb{R}^{4}$ and let [] be a regular or an ambient isotopy invariant of classical knots and links in 3-space. Let $(\mathbf{a}, \mathbf{s})=\left(a_{1}, \ldots, a_{m}, s_{1}, s_{2}, s_{3}, s_{4}\right) \in \mathbf{V}(\Delta ;[])$ such that $\alpha(\mathbf{a})$ is invertible and $\lambda(\mathbf{a}, \mathbf{s})=\lambda_{1}(\mathbf{a}, \mathbf{s}) \lambda_{2}(\mathbf{a}, \mathbf{s}) \neq 0$. Then

$$
L_{D}(\mathbf{a}, \mathbf{s})=\alpha(\mathbf{a})^{-t(D)} \lambda(\mathbf{a}, \mathbf{s})^{-|V(D)|}\langle\langle D\rangle\rangle(\mathbf{a}, \mathbf{s})
$$

is an invariant of the (stably) equivalence class of $\mathcal{L}$.

Proof. By Lemma 4.2, $J_{D}(\mathbf{a}, \mathbf{s})$ and $J_{D^{*}}(\mathbf{a}, \mathbf{s})$ are invariant under the moves $\Omega_{1}, \ldots$, $\Omega_{5}, \Omega_{7}, \Omega_{8}$ and their mirror moves and so is the product

$$
J_{D}(\mathbf{a}, \mathbf{s}) J_{D^{*}}(\mathbf{a}, \mathbf{s})=\alpha(\mathbf{a})^{-t_{+}(D)-t_{+}\left(D^{*}\right)}\langle\langle D\rangle\rangle(\mathbf{a}, \mathbf{s}) .
$$

It is immediate from the definition that $t_{+}\left(D^{*}\right)=t_{-}(D)$ and hence $-t_{+}(D)-$ $t_{+}\left(D^{*}\right)=-t_{+}(D)-t_{-}(D)=-t(D)$. On the other hand, it is clear that $|V(D)|$ is invariant under all moves $\Omega_{i}$, except the moves $\Omega_{6}$ and $\Omega_{6}^{*}$, and so is $\lambda(\mathbf{a}, \mathbf{s})^{-|V(D)|}$. Thus the product $\lambda(\mathbf{a}, \mathbf{s})^{-|V(D)|} J_{D}(\mathbf{a}, \mathbf{s}) J_{D^{*}}(\mathbf{a}, \mathbf{s})=L_{D}(\mathbf{a}, \mathbf{s})$ is invariant under all moves $\Omega_{i}$, except the moves $\Omega_{6}$ and $\Omega_{6}^{*}$. Now, by Lemma 3.4, it follows that

$$
\begin{aligned}
& \langle\langle\chi\rangle\rangle(\mathbf{a}, \mathbf{s})=[[\chi \bigcirc]](\mathbf{a}, \mathbf{s})\left[\left[\chi^{*}\right]\right](\mathbf{a}, \mathbf{s}) \\
& =[[\chi]](\mathbf{a}, \mathbf{s})[[\mathcal{X}]](\mathbf{a}, \mathbf{s}) \\
& \left.\left.=\left(\lambda_{1}(\mathbf{a}, \mathbf{s})[[]]\right](\mathbf{a}, \mathbf{s})\right)\left(\lambda_{2}(\mathbf{a}, \mathbf{s})\left[[)^{*}\right]\right](\mathbf{a}, \mathbf{s})\right) \\
& \left.\left.=\lambda_{1}(\mathbf{a}, \mathbf{s}) \lambda_{2}(\mathbf{a}, \mathbf{s})[[]]\right](\mathbf{a}, \mathbf{s})\left[[)^{*}\right]\right](\mathbf{a}, \mathbf{s}) \\
& =\lambda(\mathbf{a}, \mathbf{s})\langle\langle\rangle\rangle\rangle(\mathbf{a}, \mathbf{s}) \text {. }
\end{aligned}
$$

Similarly,

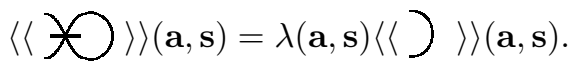


By Lemma 4.1 (1), $t(囚)=t())=t(\mathcal{X )}$ ) and hence

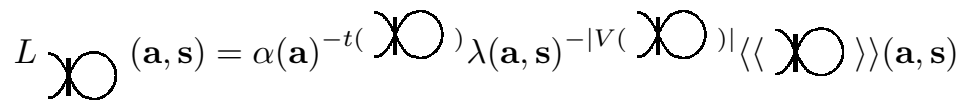

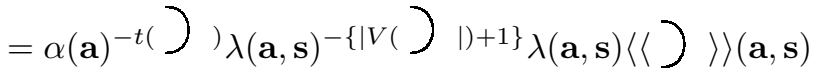

$$
\begin{aligned}
& \left.\left.\left.\left.=\alpha(\mathbf{a})^{-t(}\right){ }^{\prime} \lambda(\mathbf{a}, \mathbf{s})^{-\mid V()}\right) \|_{\langle}\right\rangle\right\rangle(\mathbf{a}, \mathbf{s}) \\
& =L)(\mathbf{a}, \mathbf{s})
\end{aligned}
$$

and

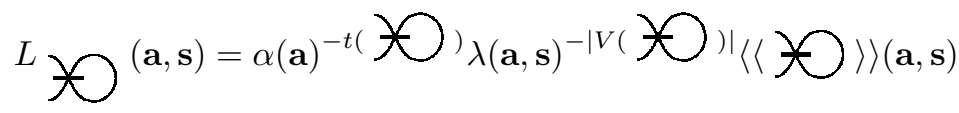

$$
\begin{aligned}
& \left.\left.=\alpha(\mathbf{a})^{-t(}\right) \lambda(\mathbf{a}, \mathbf{s})^{-\{\mid V()}\right)^{\mid+1\}} \lambda(\mathbf{a}, \mathbf{s})\langle\langle\supset\rangle\rangle(\mathbf{a}, \mathbf{s}) \\
& \left.\left.=\alpha(\mathbf{a})^{-t(}\right){ }^{\prime} \lambda(\mathbf{a}, \mathbf{s})^{-\mid V(}\right){ }^{\prime}\langle\langle\rangle\rangle(\mathbf{a}, \mathbf{s}) \\
& =L)(\mathbf{a}, \mathbf{s}) \text {. }
\end{aligned}
$$

This proves the invariance of $L_{D}(\mathbf{a}, \mathbf{s})$ under the moves $\Omega_{6}$ and $\Omega_{6}^{*}$. Finally, in the case that [ ] is an ambient isotopy invariant, taking $\alpha(\mathbf{a})=1$, the argument above easily implies that $L_{D}(\mathbf{a}, \mathbf{s})$ is invariant under all the moves $\Omega_{i}(i=1,2, \ldots, 8)$ and $\Omega_{6}^{*}$. This completes the proof.

Theorem 4.4. Let $D$ be any ch-diagram of a surface link $\mathcal{L}$ in $\mathbb{R}^{4}$ and let [ ] be a regular or an ambient isotopy invariant of classical knots and links in 3-space. Let $(\mathbf{a}, \mathbf{s})=\left(a_{1}, \ldots, a_{m}, s_{1}, s_{2}, s_{3}, s_{4}\right) \in \mathbf{V}(\Delta ;[])$ such that $\alpha(\mathbf{a})$ is invertible.

(1) If $\lambda_{1}(\mathbf{a}, \mathbf{s})=\lambda_{2}(\mathbf{a}, \mathbf{s}) \neq 0$, then

$$
J_{D}^{1}(\mathbf{a}, \mathbf{s})=\lambda_{1}(\mathbf{a}, \mathbf{s})^{-|V(D)|} J_{D}(\mathbf{a}, \mathbf{s})=\frac{[[D]](\mathbf{a}, \mathbf{s})}{\alpha(\mathbf{a})^{t_{+}(D)} \lambda_{1}(\mathbf{a}, \mathbf{s})^{|V(D)|}}
$$

is an invariant of the (stably) equivalence class of $\mathcal{L}$.

(2) If $\lambda_{2}(\mathbf{a}, \mathbf{s})=\lambda_{1}(\mathbf{a}, \mathbf{s})^{-1} \neq 1$, then

$$
J_{D}^{2}(\mathbf{a}, \mathbf{s})=\lambda_{1}(\mathbf{a}, \mathbf{s})^{-e(D)} J_{D}(\mathbf{a}, \mathbf{s})=\frac{[[D]](\mathbf{a}, \mathbf{s})}{\alpha(\mathbf{a})^{t_{+}(D)} \lambda_{1}(\mathbf{a}, \mathbf{s})^{e(D)}}
$$

is an invariant of the (stably) equivalence class of $\mathcal{L}$.

Proof. By Lemma 4.2 and Lemma 4.1(2), it is immediate that $J_{D}^{i}(\mathbf{a}, \mathbf{s}), i=1,2$, is invariant under the moves $\Omega_{1}, \ldots, \Omega_{5}, \Omega_{7}, \Omega_{8}$ and their mirror moves. Hence it remains to prove that $J_{D}^{i}(\mathbf{a}, \mathbf{s}), i=1,2$, is invariant under the moves $\Omega_{6}$ and $\Omega_{6}^{*}$. 
By Lemma 4.1(1), $t_{+}(D)$ is invariant under the moves $\Omega_{6}$ and $\Omega_{6}^{*}$ and so it follows from Lemma 3.4 and Lemma 4.1(2) that

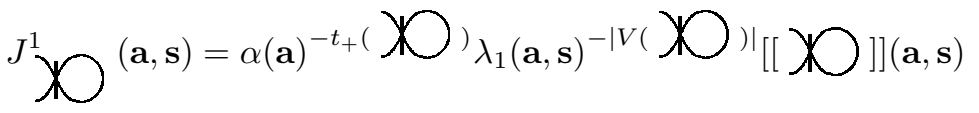

$$
\begin{aligned}
& \left.\left.\left.=\alpha(\mathbf{a})^{-t_{+}(}\right){ }^{\prime} \lambda_{1}(\mathbf{a}, \mathbf{s})^{-\{\mid V(}\right){ }^{) \mid+1\}} \lambda_{1}(\mathbf{a}, \mathbf{s})[[]]\right](\mathbf{a}, \mathbf{s})
\end{aligned}
$$

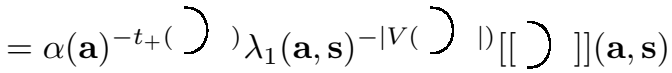

$$
\begin{aligned}
& =J^{1}(\mathbf{a}, \mathbf{s})
\end{aligned}
$$

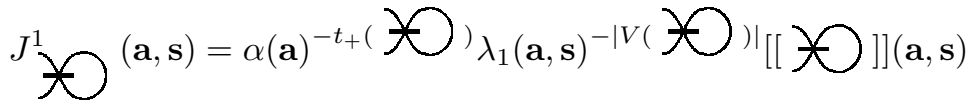

$$
\begin{aligned}
& \left.\left.\left.=\alpha(\mathbf{a})^{-t_{+}(}\right){ }^{\prime} \lambda_{1}(\mathbf{a}, \mathbf{s})^{-\{\mid V()}\right)^{\prime+1\}} \lambda_{2}(\mathbf{a}, \mathbf{s})[[]]\right](\mathbf{a}, \mathbf{s}) \\
& \left.\left.\left.=\alpha(\mathbf{a})^{-t_{+}(}\right){ }^{)} \lambda_{1}(\mathbf{a}, \mathbf{s})^{-\{\mid V()}\right)^{{ }^{+1+1\}}} \lambda_{1}(\mathbf{a}, \mathbf{s})[[]]\right](\mathbf{a}, \mathbf{s}) \\
& =\alpha(\mathbf{a})^{-t_{+}}(){ }^{)} \lambda_{1}(\mathbf{a}, \mathbf{s})^{-\mid V()) \mid}[[]][(\mathbf{a}, \mathbf{s}) \\
& =J^{1}(\mathbf{a}, \mathbf{s}) \text {, }
\end{aligned}
$$

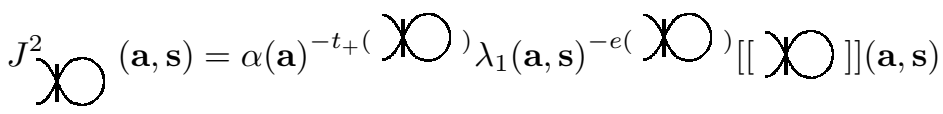

$$
\begin{aligned}
& \left.\left.\left.=\alpha(\mathbf{a})^{-t_{+}(}\right){ }^{)} \lambda_{1}(\mathbf{a}, \mathbf{s})^{-\{e(}\right){ }^{)+1\}} \lambda_{1}(\mathbf{a}, \mathbf{s})[[]]\right](\mathbf{s})
\end{aligned}
$$

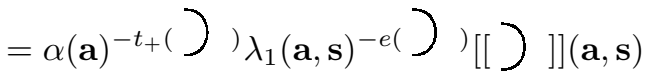

$$
\begin{aligned}
& =J^{2}(\mathbf{a}, \mathbf{s})
\end{aligned}
$$

and

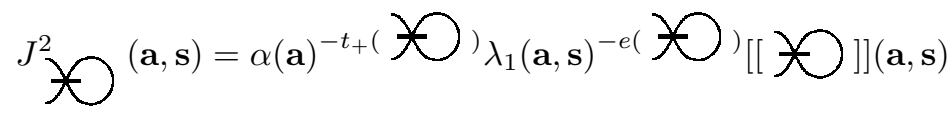

$$
\begin{aligned}
& \left.\left.=\alpha(\mathbf{a})^{-t_{+}(}\right){ }^{)} \lambda_{1}(\mathbf{a}, \mathbf{s})^{-\{e(}{ }^{-1\}} \lambda_{2}(\mathbf{a}, \mathbf{s})[[]]\right](\mathbf{a}, \mathbf{s}) \\
& \left.\left.=\alpha(\mathbf{a})^{-t_{+}()}{ }^{\prime} \lambda_{1}(\mathbf{a}, \mathbf{s})^{-\{e(}\right){ }^{-1\}} \lambda_{1}(\mathbf{a}, \mathbf{s})^{-1}[[]]\right](\mathbf{a}, \mathbf{s})
\end{aligned}
$$

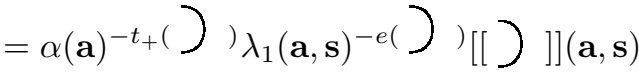

$$
\begin{aligned}
& =J^{2}(\mathbf{a}, \mathbf{s}) \text {. }
\end{aligned}
$$

This proves the invariance of $J_{D}^{i}(\mathbf{a}, \mathbf{s}), i=1,2$, under the moves $\Omega_{6}$ and $\Omega_{6}^{*}$. Finally, in the case that [ ] is an ambient isotopy invariant, taking $\alpha(\mathbf{a})=1$, the argument above easily implies that $J_{D}^{i}(\mathbf{a}, \mathbf{s})$ is invariant under all moves $\Omega_{i}(i=1,2, \ldots, 8)$ and $\Omega_{6}^{*}$. This completes the proof. 
Remark 4.5. In the formulas (4.5), (4.7) and (4.8), we may use $t_{-}(D)$ or $\frac{t(D)}{2}$ instead of $t_{+}(D)$.

It is natural to ask which (ambient or regular isotopy) invariants of classical knots and links give rise to non-trivial invariants of equivalent surface links in $\mathbb{R}^{4}$ in the formalisms of Theorems 4.3 or 4.4 In the next section 5, we shall discuss this question with Kauffman's bracket polynomial [13, which gives an affirmative answer.

\section{The InVARIANTS VIA THE BRACKET POLYNOMIAL}

Let $K$ be a classical knot or link diagram. The Kauffman bracket polynomial of $K\left[13\right.$ is a Laurent polynomial $\langle K\rangle=\langle K\rangle(A) \in \hat{R}=\mathbb{Z}\left[A, A^{-1}\right]$ defined by the following rules:

$(B 1)\langle\bigcirc\rangle=1$

(B2) $\left\langle\bigcirc K^{\prime}\right\rangle=\delta\left\langle K^{\prime}\right\rangle$, where $\delta=\delta(A)=-A^{2}-A^{-2}$,

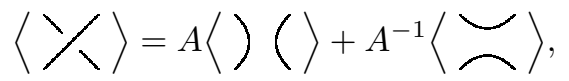

where $\bigcirc K^{\prime}$ denotes any addition of a disjoint circle $\bigcirc$ to a knot or link diagram $K^{\prime}$. Note that the Kauffman bracket polynomial is not an ambient isotopy invariant, but it is a regular isotopy invariant and, for $\alpha=\alpha(A)=-A^{3}$,

$$
\left.\left.\langle\mathcal{O}\rangle=-A^{3}\langle\rangle\right\rangle,\langle\lambda \bigcirc\rangle=-A^{-3}\langle\rangle\right\rangle
$$

For the rest of the paper, we mean by $[[D]]$ the polynomial $[[D]](A, x, y, z, w)$ in Definition 3.1 with [ ] $=\langle\rangle(A)$, the Kauffman bracket polynomial, and by $\mathbb{E}=\mathbb{C}(A, x, y, z, w)$, the rational function field of $\mathbb{C}[A, x, y, z, w]$, unless otherwise specified. By (L2) of Definition 3.1, it follows that

$$
\begin{aligned}
& {[[\nless]]=[[\smile]] x+[[久]] y+[[久]] z+[[)(]] w}
\end{aligned}
$$

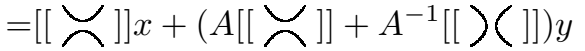

$$
\begin{aligned}
& +\left(A[[)(]]+A^{-1}\left[\left[\smile_{\smile}\right]\right]\right) z+[[)(]] w
\end{aligned}
$$

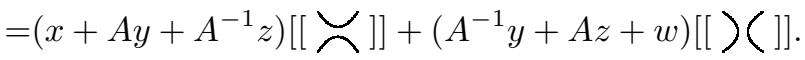

This shows that $[[D]]$ is given by means of the following two rules:

(L1b) $[[D]]=\langle D\rangle(A)$ if $D$ is a classical knot or link diagram,

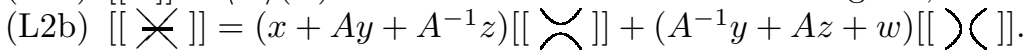

From this, one can easily see that the state-sum formula of $[[D]]$ is given by

$$
[[D]](A, x, y, z, w)=\sum_{\sigma \in \mathcal{S}_{\infty, 0}(D)}\left(x+A y+\frac{z}{A}\right)^{\sigma(\infty)}\left(\frac{y}{A}+A z+w\right)^{\sigma(0)}\left\langle D_{\sigma}\right\rangle(A) .
$$

Similarly, we have the formula:

$$
\left[\left[D^{*}\right]\right](A, x, y, z, w)=\sum_{\sigma \in \mathcal{S}_{\infty, 0}(D)}\left(x+A y+\frac{z}{A}\right)^{\sigma(\infty)}\left(\frac{y}{A}+A z+w\right)^{\sigma(0)}\left\langle D_{\sigma^{*}}\right\rangle(A),
$$


where $\mathcal{S}_{\infty, 0}(D)$ is the set of all states of a ch-diagram $D$ which assign all marked vertices with $T_{\infty}$ and $T_{0}$ and $\sigma^{*}$ is the state of $D$ obtained from $\sigma \in \mathcal{S}(D)$ by replacing the assignment $T_{0}$ with $T_{\infty}$ and vice versa.

Example 5.1. Let $D_{1}, D_{2}$ and $D_{3}$ denote the ch-diagrams of $0_{1}, 2_{1}^{-1}$ and $2_{1}^{1}$ in Yoshikawa's table, respectively. From Example 3.2, we obtain

$$
\begin{aligned}
{\left[\left[D_{1}\right]\right] } & =1, \\
{\left[\left[D_{2}\right]\right] } & =-A^{3} x+\left(-A^{4}-A^{-4}\right) y+\left(-A^{2}-A^{-2}\right) z-A^{-3} w, \\
{\left[\left[D_{3}\right]\right] } & =x^{2}-2 A^{-3} x y-2 A^{3} x z-2 A^{3} y w-2 A^{-3} z w+w^{2} \\
& +\left(-A^{2}-A^{-2}\right)\left(y^{2}+z^{2}+2 x w\right)+2 y z\left(-A^{4}-A^{-4}\right) .
\end{aligned}
$$

On the other hand, $\left[\left[D_{1}^{*}\right]\right]=\left[\left[D_{1}\right]\right],\left[\left[D_{3}^{*}\right]\right]=\left[\left[D_{3}\right]\right]$, and $\left[\left[D_{2}^{*}\right]\right]=-A^{-3} x+$ $\left(-A^{2}-A^{-2}\right) y+\left(-A^{4}-A^{-4}\right) z-A^{3} w$. Hence

$$
\begin{aligned}
\left\langle\left\langle D_{1}\right\rangle\right\rangle(\varsigma, x, y, z, w) & =1, \\
\left\langle\left\langle D_{2}\right\rangle\right\rangle(\varsigma, x, y, z, w) & =A^{-8}\left(A^{7} x+\left(A^{6}+A^{2}\right) z+A w+\left(A^{8}+1\right) y\right) \\
& \times\left(A^{7} w+\left(A^{6}+A^{2}\right) y+A x+\left(A^{8}+1\right) z\right), \\
\left\langle\left\langle D_{3}\right\rangle\right\rangle(\varsigma, x, y, z, w) & =\left[\left[D_{3}\right]\right](\varsigma, x, y, z, w)^{2} .
\end{aligned}
$$

Let $\mathcal{F} \mathcal{T}_{n}^{c}$ denote the free $\mathbb{Z}\left[A, A^{-1}\right]$-module of all formal linear combinations of classical $n$-tangles in $\mathcal{T}_{n}^{c}$. Let $\langle\rangle:, \mathcal{T}_{n}^{c} \times \mathcal{T}_{n}^{c} \rightarrow \mathbb{Z}\left[A, A^{-1}\right]$ denote the pairing defined by the formula $\langle x, y\rangle=\langle x \circ y\rangle$, i.e., the Kauffman bracket polynomial of the knot or link diagram $x \circ y$ for all $x, y \in \mathcal{T}_{n}^{c}$. Then this pairing is naturally extended to a bilinear form $\langle\rangle:, \mathcal{F} \mathcal{T}_{n}^{c} \times \mathcal{F} \mathcal{T}_{n}^{c} \rightarrow \mathbb{Z}\left[A, A^{-1}\right]$ on the module $\mathcal{F} \mathcal{T}_{n}^{c}$. The following identities in the Temperley-Lieb algebra $V_{m}$ with generators $e_{i}, 1 \leq i \leq m-1$, are useful in the sequel:

$$
\begin{aligned}
& e_{i}^{2}=\delta e_{i}(1 \leq i \leq m-1), \text { where } \delta=\delta(A)=-A^{2}-A^{-2}, \\
& e_{i} e_{i+1} e_{i}=e_{i}(1 \leq i \leq m-2), e_{i} e_{i-1} e_{i}=e_{i}(2 \leq i \leq m-1), \\
& e_{i} e_{j}=e_{j} e_{i}(|i-j|>1) .
\end{aligned}
$$

Let $M(A)=\left[a_{i j}\right]_{0 \leq i, j \leq 4}$ be the $5 \times 5$ matrix with $a_{i j}=\left\langle f_{i} \circ f_{j}\right\rangle$ and let $N(A)=$ $\left[b_{i j}\right]_{0 \leq i, j \leq 13}$ be the $14 \times 14$ matrix defined by $b_{i j}=\left\langle g_{i} \circ g_{j}\right\rangle$. Then

$$
M(A)=\left[\begin{array}{ccccc}
\delta^{2} & \delta & \delta & 1 & 1 \\
\delta & 1 & \delta^{2} & \delta & \delta \\
\delta & \delta^{2} & 1 & \delta & \delta \\
1 & \delta & \delta & 1 & \delta^{2} \\
1 & \delta & \delta & \delta^{2} & 1
\end{array}\right]
$$


and

$$
N(A)=\left[\begin{array}{cccccccccccccc}
\delta^{3} & \delta^{2} & \delta^{2} & \delta^{2} & \delta & \delta & \delta & \delta & \delta & 1 & 1 & 1 & 1 & \delta \\
\delta^{2} & \delta & \delta & \delta^{3} & 1 & \delta^{2} & 1 & \delta^{2} & \delta^{2} & \delta & \delta & \delta & \delta & 1 \\
\delta^{2} & \delta & \delta^{3} & \delta & \delta^{2} & 1 & \delta^{2} & \delta^{2} & \delta^{2} & \delta & \delta & \delta & \delta & \delta^{2} \\
\delta^{2} & \delta^{3} & \delta & \delta & \delta^{2} & \delta^{2} & \delta^{2} & 1 & 1 & \delta & \delta & \delta & \delta & 1 \\
\delta & 1 & \delta^{2} & \delta^{2} & \delta & \delta & \delta & \delta & \delta^{3} & 1 & \delta^{2} & 1 & \delta^{2} & \delta \\
\delta & \delta^{2} & 1 & \delta^{2} & \delta & \delta^{3} & \delta & \delta & \delta & \delta^{2} & \delta^{2} & \delta^{2} & \delta^{2} & \delta \\
\delta & 1 & \delta^{2} & \delta^{2} & \delta & \delta & \delta & \delta^{3} & \delta & \delta^{2} & 1 & \delta^{2} & 1 & \delta \\
\delta & \delta^{2} & \delta^{2} & 1 & \delta & \delta & \delta^{3} & \delta & \delta & 1 & 1 & \delta^{2} & \delta^{2} & \delta \\
\delta & \delta^{2} & \delta^{2} & 1 & \delta^{3} & \delta & \delta & \delta & \delta & \delta^{2} & \delta^{2} & 1 & 1 & \delta \\
1 & \delta & \delta & \delta & 1 & \delta^{2} & \delta^{2} & 1 & \delta^{2} & \delta & \delta & \delta & \delta^{3} & 1 \\
1 & \delta & \delta & \delta & \delta^{2} & \delta^{2} & 1 & 1 & \delta^{2} & \delta & \delta^{3} & \delta & \delta & \delta^{2} \\
1 & \delta & \delta & \delta & 1 & \delta^{2} & \delta^{2} & \delta^{2} & 1 & \delta & \delta & \delta^{3} & \delta & \delta^{2} \\
1 & \delta & \delta & \delta & \delta^{2} & \delta^{2} & 1 & \delta^{2} & 1 & \delta^{3} & \delta & \delta & \delta & 1 \\
\delta & 1 & \delta^{2} & 1 & \delta & \delta & \delta & \delta & \delta & 1 & \delta^{2} & \delta^{2} & 1 & \delta^{3}
\end{array}\right]
$$

Lemma 5.2. Let $U$ be any classical 3-tangle diagram in $\mathcal{T}_{3}^{c}$. Then

$$
\Delta_{7}(A, x, y, z, w ; U)=\left(\delta^{2}-1\right) \chi(A, x, y, z, w) \phi_{U}(A)
$$

where $\chi(A, x, y, z, w)=A^{-2}\left(w A+y+z A^{2}\right)\left(y A^{2}+x A+z\right)$ and $\phi_{U}(A)$ is a Laurent polynomial in $\mathbb{Z}\left[A, A^{-1}\right]$.

Proof. We first observe that for any classical 3-tangle diagram $J \in \mathcal{T}_{3}^{c}$, nullifying all classical crossings of $U$ by applying the Kauffman bracket polynomial axioms $\left(B_{i}\right), i=1,2,3$, we have

$$
\langle J \circ U\rangle=\sum_{i=0}^{4} \phi_{U}^{i}(A)\left\langle J \circ f_{i}\right\rangle,
$$

where $\phi_{U}^{i}(A) \in \mathbb{Z}\left[A, A^{-1}\right], i=0,1,2,3,4$. From (3.3) and (5.5), we obtain

$$
\begin{aligned}
& \Delta_{7}(A, x, y, z, w ; U)=\sum_{i=0}^{4} \phi_{U}^{i}(A)\left(x w\left(\left\langle f_{4} \circ f_{i}\right\rangle-\left\langle f_{3} \circ f_{i}\right\rangle\right)\right. \\
& \quad+z w\left(\left\langle f_{2} \sigma_{1} \circ f_{i}\right\rangle-\left\langle\sigma_{1} f_{2} \circ f_{i}\right\rangle\right)+y w\left(\left\langle f_{2} \sigma_{1}^{-1} \circ f_{i}\right\rangle-\left\langle\sigma_{1}^{-1} f_{2} \circ f_{i}\right\rangle\right) \\
& \quad+x y\left(\left\langle\sigma_{2} f_{1} \circ f_{i}\right\rangle-\left\langle f_{1} \sigma_{2} \circ f_{i}\right\rangle\right)+x z\left(\left\langle\sigma_{2}^{-1} f_{1} \circ f_{i}\right\rangle-\left\langle f_{1} \sigma_{2}^{-1} \circ f_{i}\right\rangle\right) \\
& \quad+y^{2}\left(\left\langle\sigma_{2} \sigma_{1}^{-1} \circ f_{i}\right\rangle-\left\langle\sigma_{1}^{-1} \sigma_{2} \circ f_{i}\right\rangle\right)+z^{2}\left(\left\langle\sigma_{2}^{-1} \sigma_{1} \circ f_{i}\right\rangle-\left\langle\sigma_{1} \sigma_{2}^{-1} \circ f_{i}\right\rangle\right) \\
& \left.\quad+y z\left(\left\langle\sigma_{2} \sigma_{1} \circ f_{i}\right\rangle-\left\langle\sigma_{1} \sigma_{2} \circ f_{i}\right\rangle+\left\langle\sigma_{2}^{-1} \sigma_{1}^{-1} \circ f_{i}\right\rangle-\left\langle\sigma_{1}^{-1} \sigma_{2}^{-1} \circ f_{i}\right\rangle\right)\right) \\
& =\sum_{i=0}^{4} \phi_{U}^{i}(A) \Delta_{7}\left(A, x, y, z, w ; f_{i}\right) .
\end{aligned}
$$


By a straightforward computation, we obtain that

$$
\begin{aligned}
& \left\langle f_{2} \sigma_{1}, f_{i}\right\rangle=A\left\langle f_{2}, f_{i}\right\rangle+A^{-1}\left\langle f_{4}, f_{i}\right\rangle, \\
& \left\langle\sigma_{1} f_{2}, f_{i}\right\rangle=A\left\langle f_{2}, f_{i}\right\rangle+A^{-1}\left\langle f_{3}, f_{i}\right\rangle, \\
& \left\langle f_{2} \sigma_{1}^{-1}, f_{i}\right\rangle=A^{-1}\left\langle f_{2}, f_{i}\right\rangle+A\left\langle f_{4}, f_{i}\right\rangle, \\
& \left\langle\sigma_{1}^{-1} f_{2}, f_{i}\right\rangle=A^{-1}\left\langle f_{2}, f_{i}\right\rangle+A\left\langle f_{3}, f_{i}\right\rangle, \\
& \left\langle\sigma_{2} f_{1}, f_{i}\right\rangle=A\left\langle f_{1}, f_{i}\right\rangle+A^{-1}\left\langle f_{4}, f_{i}\right\rangle, \\
& \left\langle f_{1} \sigma_{2}, f_{i}\right\rangle=A\left\langle f_{1}, f_{i}\right\rangle+A^{-1}\left\langle f_{3}, f_{i}\right\rangle, \\
& \left\langle\sigma_{2}^{-1} f_{1}, f_{i}\right\rangle=A^{-1}\left\langle f_{1}, f_{i}\right\rangle+A\left\langle f_{4}, f_{i}\right\rangle, \\
& \left\langle f_{1} \sigma_{2}^{-1}, f_{i}\right\rangle=A^{-1}\left\langle f_{1}, f_{i}\right\rangle+A\left\langle f_{3}, f_{i}\right\rangle, \\
& \left\langle\sigma_{2} \sigma_{1}^{-1}, f_{i}\right\rangle=\left\langle f_{0}, f_{i}\right\rangle+A^{2}\left\langle f_{1}, f_{i}\right\rangle+A^{-2}\left\langle f_{2}, f_{i}\right\rangle+\left\langle f_{4}, f_{i}\right\rangle, \\
& \left\langle\sigma_{1}^{-1} \sigma_{2}, f_{i}\right\rangle=\left\langle f_{0}, f_{i}\right\rangle+A^{2}\left\langle f_{1}, f_{i}\right\rangle+A^{-2}\left\langle f_{2}, f_{i}\right\rangle+\left\langle f_{3}, f_{i}\right\rangle, \\
& \left\langle\sigma_{2}^{-1} \sigma_{1}, f_{i}\right\rangle=\left\langle f_{0}, f_{i}\right\rangle+A^{-2}\left\langle f_{1}, f_{i}\right\rangle+A^{2}\left\langle f_{2}, f_{i}\right\rangle+\left\langle f_{4}, f_{i}\right\rangle, \\
& \left\langle\sigma_{1} \sigma_{2}^{-1}, f_{i}\right\rangle=\left\langle f_{0}, f_{i}\right\rangle+A^{-2}\left\langle f_{1}, f_{i}\right\rangle+A^{2}\left\langle f_{2}, f_{i}\right\rangle+\left\langle f_{3}, f_{i}\right\rangle, \\
& \left\langle\sigma_{2} \sigma_{1}, f_{i}\right\rangle=A^{2}\left\langle f_{0}, f_{i}\right\rangle+\left\langle f_{1}, f_{i}\right\rangle+\left\langle f_{2}, f_{i}\right\rangle+A^{-2}\left\langle f_{4}, f_{i}\right\rangle, \\
& \left\langle\sigma_{1} \sigma_{2}, f_{i}\right\rangle=A^{2}\left\langle f_{0}, f_{i}\right\rangle+\left\langle f_{1}, f_{i}\right\rangle+\left\langle f_{2}, f_{i}\right\rangle+A^{-2}\left\langle f_{3}, f_{i}\right\rangle, \\
& \left\langle\sigma_{2}^{-1} \sigma_{1}^{-1}, f_{i}\right\rangle=A^{-2}\left\langle f_{0}, f_{i}\right\rangle+\left\langle f_{1}, f_{i}\right\rangle+\left\langle f_{2}, f_{i}\right\rangle+A^{2}\left\langle f_{4}, f_{i}\right\rangle, \\
& \left\langle\sigma_{1}^{-1} \sigma_{2}^{-1}, f_{i}\right\rangle=A^{-2}\left\langle f_{0}, f_{i}\right\rangle+\left\langle f_{1}, f_{i}\right\rangle+\left\langle f_{2}, f_{i}\right\rangle+A^{2}\left\langle f_{3}, f_{i}\right\rangle .
\end{aligned}
$$

From these computations together with the matrix $M(A)$ in (5.3), we obtain

$$
\begin{aligned}
\Delta_{7}\left(A, x, y, z, w ; f_{i}\right)= & \left(x w+z w A^{-1}+y w A+x y A^{-1}+x z A+y^{2}+z^{2}\right. \\
& \left.+y z\left(A^{-2}+A^{2}\right)\right)\left(\left\langle f_{4}, f_{i}\right\rangle-\left\langle f_{3}, f_{i}\right\rangle\right) \\
= & \chi(A, x, y, z, w)\left(\left\langle f_{4}, f_{i}\right\rangle-\left\langle f_{3}, f_{i}\right\rangle\right) \\
= & \begin{cases}0, & i=0,1,2 ; \\
\left(\delta^{2}-1\right) \chi(A, x, y, z, w), & i=3 ; \\
\left(1-\delta^{2}\right) \chi(A, x, y, z, w), & i=4 .\end{cases}
\end{aligned}
$$

From (5.6), it follows that

$$
\begin{aligned}
\Delta_{7}(A, x, y, z, w ; U) & =\phi_{U}^{3}(A) \Delta_{7}\left(A, x, y, z, w ; f_{3}\right)+\phi_{U}^{4}(A) \Delta_{7}\left(A, x, y, z, w ; f_{4}\right) \\
& =\left(\delta^{2}-1\right) \chi(A, x, y, z, w)\left(\phi_{U}^{3}(A)-\phi_{U}^{4}(A)\right) \\
& =\left(\delta^{2}-1\right) \chi(A, x, y, z, w) \phi_{U}(A),
\end{aligned}
$$

where $\phi_{U}(A)=\phi_{U}^{3}(A)-\phi_{U}^{4}(A) \in \mathbb{Z}\left[A, A^{-1}\right]$. This completes the proof of Lemma 5.2 .

Lemma 5.3 ([16, Lemma 4.2]). Let $\beta=\sigma_{2} \sigma_{1} \sigma_{3} \sigma_{2} \in B_{4}$ and let $\beta^{*}=$ $\sigma_{2}^{-1} \sigma_{1}^{-1} \sigma_{3}^{-1} \sigma_{2}^{-1} \in B_{4}$. Then

(1) $\left\langle\beta, g_{0}\right\rangle=-A^{10}-A^{2},\left\langle\beta, g_{13}\right\rangle=-A^{-10}-A^{-2}$,

(2) For each $j=1,2, \ldots, 12,\left\langle\beta, g_{j}\right\rangle=\left\langle\beta^{*}, g_{j}\right\rangle$, and $\left\langle\beta^{*}, g_{0}\right\rangle=\left\langle\beta, g_{13}\right\rangle$.

(3) For each $j=0,1, \ldots, 13,\left\langle g_{1} \beta, g_{j}\right\rangle=\left\langle g_{1} \beta^{*}, g_{j}\right\rangle,\left\langle g_{3} \beta, g_{j}\right\rangle=\left\langle g_{3} \beta^{*}, g_{j}\right\rangle$, and $\left\langle g_{5} \beta, g_{j}\right\rangle=\left\langle g_{5} \beta^{*}, g_{j}\right\rangle$. 
Lemma 5.4. Let $V$ be any classical 4-tangle diagram in $\mathcal{T}_{4}^{c}$. Then

$\Delta_{8}(A, x, y, z, w ; V)=\left(\delta^{2}-1\right) \chi(A, x, y, z, w)\left(A^{6}-A^{2}+A^{-2}-A^{-6}\right) \psi_{V}(A)$, for some Laurent polynomial $\psi_{V}(A) \in \mathbb{Z}\left[A, A^{-1}\right]$.

Proof. Note that for any classical 4-tangle $J \in \mathcal{T}_{4}^{c}$, it follows that

$$
\langle J \circ V\rangle=\sum_{j=0}^{13} \phi_{V}^{j}(A)\left\langle J \circ g_{j}\right\rangle,
$$

where $\psi_{V}^{j}(A) \in \mathbb{Z}\left[A, A^{-1}\right], j=0,1,2, \ldots, 13$. From (3.4) and (5.7),

$$
\begin{aligned}
& \Delta_{8}(A, x, y, z, w ; V)=\sum_{i=0}^{13} \psi_{V}^{j}(A)\left(x w\left(\left[\beta \circ g_{j}\right]-\left[\beta^{*} \circ g_{j}\right]\right)\right. \\
& \quad+y w\left(\left[\sigma_{1} \beta \circ g_{j}\right]-\left[\sigma_{1} \beta^{*} \circ g_{j}\right]\right)+z w\left(\left[\sigma_{1}^{-1} \beta \circ g_{j}\right]-\left[\sigma_{1}^{-1} \beta^{*} \circ g_{j}\right]\right) \\
& \left.\quad+y z\left(\left[\sigma_{1} \sigma_{3} \beta \circ g_{j}\right]-\left[\sigma_{1} \sigma_{3} \beta^{*} \circ g_{j}\right]\right)+\left[\sigma_{1}^{-1} \sigma_{3}^{-1} \beta \circ g_{j}\right]-\left[\sigma_{1}^{-1} \sigma_{3}^{-1} \beta^{*} \circ g_{j}\right]\right) \\
& \quad+y^{2}\left(\left[\sigma_{1} \sigma_{3}^{-1} \beta \circ g_{j}\right]-\left[\sigma_{1} \sigma_{3}^{-1} \beta^{*} \circ g_{j}\right]\right)+z^{2}\left(\left[\sigma_{1}^{-1} \sigma_{3} \beta \circ g_{j}\right]-\left[\sigma_{1}^{-1} \sigma_{3} \beta^{*} \circ g_{j}\right]\right) \\
& \left.\quad+x z\left(\left[\sigma_{3} \beta \circ g_{j}\right]-\left[\sigma_{3} \beta^{*} \circ g_{j}\right]\right)+x y\left(\left[\sigma_{3}^{-1} \beta \circ g_{j}\right]-\left[\sigma_{3}^{-1} \beta^{*} \circ g_{j}\right]\right)\right) \\
& =\sum_{i=0}^{13} \psi_{V}^{j}(A) \Delta_{8}\left(A, x, y, z, w ; g_{j}\right) .
\end{aligned}
$$

By a straightforward computation, we obtain

$$
\begin{aligned}
& \left\langle\sigma_{1} \beta, g_{j}\right\rangle=A\left\langle\beta, g_{j}\right\rangle+A^{-1}\left\langle g_{1} \beta, g_{j}\right\rangle, \\
& \left\langle\sigma_{1} \beta^{*}, g_{j}\right\rangle=A\left\langle\beta^{*}, g_{j}\right\rangle+A^{-1}\left\langle g_{1} \beta^{*}, g_{j}\right\rangle, \\
& \left\langle\sigma_{1}^{-1} \beta, g_{j}\right\rangle=A^{-1}\left\langle\beta, g_{j}\right\rangle+A\left\langle g_{1} \beta, g_{j}\right\rangle, \\
& \left\langle\sigma_{1}^{-1} \beta^{*}, g_{j}\right\rangle=A^{-1}\left\langle\beta^{*}, g_{j}\right\rangle+A\left\langle g_{1} \beta^{*}, g_{j}\right\rangle, \\
& \left\langle\sigma_{1} \sigma_{3} \beta, g_{j}\right\rangle=A^{2}\left\langle\beta, g_{j}\right\rangle+\left\langle g_{1} \beta, g_{j}\right\rangle+\left\langle g_{3} \beta, g_{j}\right\rangle+A^{-2}\left\langle g_{5} \beta, g_{j}\right\rangle, \\
& \left\langle\sigma_{1} \sigma_{3} \beta^{*}, g_{j}\right\rangle=A^{2}\left\langle\beta^{*}, g_{j}\right\rangle+\left\langle g_{1} \beta^{*}, g_{j}\right\rangle+\left\langle g_{3} \beta^{*}, g_{j}\right\rangle+A^{-2}\left\langle g_{5} \beta^{*}, g_{j}\right\rangle, \\
& \left\langle\sigma_{1}^{-1} \sigma_{3}^{-1} \beta, g_{j}\right\rangle=A^{-2}\left\langle\beta, g_{j}\right\rangle+\left\langle g_{1} \beta, g_{j}\right\rangle+\left\langle g_{3} \beta, g_{j}\right\rangle+A^{2}\left\langle g_{5} \beta, g_{j}\right\rangle, \\
& \left\langle\sigma_{1}^{-1} \sigma_{3}^{-1} \beta^{*}, g_{j}\right\rangle=A^{-2}\left\langle\beta^{*}, g_{j}\right\rangle+\left\langle g_{1} \beta^{*}, g_{j}\right\rangle+\left\langle g_{3} \beta^{*}, g_{j}\right\rangle+A^{2}\left\langle g_{5} \beta^{*}, g_{j}\right\rangle, \\
& \left\langle\sigma_{1} \sigma_{3}^{-1} \beta, g_{j}\right\rangle=\left\langle\beta, g_{j}\right\rangle+A^{-2}\left\langle g_{1} \beta, g_{j}\right\rangle+A^{2}\left\langle g_{3} \beta, g_{j}\right\rangle+\left\langle g_{5} \beta, g_{j}\right\rangle, \\
& \left\langle\sigma_{1} \sigma_{3}^{-1} \beta^{*}, g_{j}\right\rangle=\left\langle\beta^{*}, g_{j}\right\rangle+A^{-2}\left\langle g_{1} \beta^{*}, g_{j}\right\rangle+A^{2}\left\langle g_{3} \beta^{*}, g_{j}\right\rangle+\left\langle g_{5} \beta^{*}, g_{j}\right\rangle, \\
& \left\langle\sigma_{1}^{-1} \sigma_{3} \beta, g_{j}\right\rangle=\left\langle\beta, g_{j}\right\rangle+A^{2}\left\langle g_{1} \beta, g_{j}\right\rangle+A^{-2}\left\langle g_{3} \beta, g_{j}\right\rangle+\left\langle g_{5} \beta, g_{j}\right\rangle, \\
& \left\langle\sigma_{1}^{-1} \sigma_{3} \beta^{*}, g_{j}\right\rangle=\left\langle\beta^{*}, g_{j}\right\rangle+A^{2}\left\langle g_{1} \beta^{*}, g_{j}\right\rangle+A^{-2}\left\langle g_{3} \beta^{*}, g_{j}\right\rangle+\left\langle g_{5} \beta^{*}, g_{j}\right\rangle, \\
& \left\langle\sigma_{3} \beta, g_{j}\right\rangle=A\left\langle\beta, g_{j}\right\rangle+A^{-1}\left\langle g_{3} \beta, g_{j}\right\rangle, \\
& \left\langle\sigma_{3} \beta^{*}, g_{j}\right\rangle=A\left\langle\beta^{*}, g_{j}\right\rangle+A^{-1}\left\langle g_{3} \beta^{*}, g_{j}\right\rangle, \\
& \left\langle\sigma_{3}^{-1} \beta, g_{j}\right\rangle=A^{-1}\left\langle\beta, g_{j}\right\rangle+A\left\langle g_{3} \beta, g_{j}\right\rangle, \\
& \left\langle\sigma_{3}^{-1} \beta^{*}, g_{j}\right\rangle=A^{-1}\left\langle\beta^{*}, g_{j}\right\rangle+A\left\langle g_{3} \beta^{*}, g_{j}\right\rangle .
\end{aligned}
$$


From these computations together with Lemma 5.3 we obtain

$$
\begin{aligned}
& \Delta_{8}\left(A, x, y, z, w ; g_{j}\right) \\
& =\left(x w+y w A+\frac{z w}{A}-\delta y z+y^{2}+z^{2}+x z A+\frac{x y}{A}\right)\left(\left\langle\beta, g_{j}\right\rangle-\left\langle\beta^{*}, g_{j}\right\rangle\right) \\
& =\chi(A, x, y, z, w)\left(\left\langle\beta, g_{j}\right\rangle-\left\langle\beta^{*}, g_{j}\right\rangle\right) \\
& = \begin{cases}0, & \text { if } 1 \leq j \leq 12 ; \\
\chi(A, x, y, z, w)\left(-A^{10}-A^{2}+A^{-10}+A^{-2}\right), & \text { if } j=0 ; \\
-\chi(A, x, y, z, w)\left(-A^{10}-A^{2}+A^{-10}+A^{-2}\right), & \text { if } j=13 .\end{cases}
\end{aligned}
$$

From (5.8), we thus obtain

$$
\begin{aligned}
& \Delta_{8}(A, x, y, z, w ; V) \\
& =\psi_{V}^{0}(A) \Delta_{8}\left(A, x, y, z, w ; g_{0}\right)+\phi_{V}^{13}(A) \Delta_{8}\left(A, x, y, z, w ; g_{13}\right) \\
& =\left(-A^{10}-A^{2}+A^{-10}+A^{-2}\right) \chi(A, x, y, z, w)\left(\phi_{V}^{0}(A)-\phi_{V}^{13}(A)\right) \\
& =\left(\delta^{2}-1\right)\left(A^{6}-A^{2}+A^{-2}-A^{-6}\right) \chi(A, x, y, z, w) \psi_{V}(A),
\end{aligned}
$$

where $\psi_{V}(A)=\phi_{V}^{13}(A)-\phi_{V}^{0}(A) \in \mathbb{Z}\left[A, A^{-1}\right]$. This completes the proof.

\section{Theorem 5.5.}

$$
\begin{aligned}
& \mathbf{V}(\Delta ;\langle\rangle)=\left\{(\varsigma, x, y, z, w),(\zeta, x, y, z, w),\left(A, x, y,-A x-A^{2} y, w\right),\right. \\
&\left.\left(A, x,-A^{2} z-A w, z, w\right) \mid-\zeta^{2}-\zeta^{-2}=1,-\varsigma^{2}-\varsigma^{-2}=-1\right\} .
\end{aligned}
$$

Proof. From Lemmas 5.2 and 5.4 the result follows at once.

From now on, we investigate the invariants $L_{D}(\mathbf{a}, \mathbf{s})$ and $J_{D}^{i}(\mathbf{a}, \mathbf{s})(i=1,2)$ derived from Theorem 4.3 and Theorem 4.4 with $(\mathbf{a}, \mathbf{s}) \in \mathbf{V}(\Delta ;\langle\rangle)$. We first observe that $-\zeta^{2}-\zeta^{-2}=1$ implies that $\zeta^{3}=-\left(\zeta+\zeta^{-1}\right)=\zeta^{-3}, \delta(\zeta)=1$ and $\zeta^{3}=-1$ or 1 . Also, $-\varsigma^{2}-\varsigma^{-2}=-1$ implies that $\varsigma^{3}=\varsigma-\varsigma^{-1}=-\varsigma^{-3}, \delta(\varsigma)=-1$ and $\varsigma^{3}=\sqrt{-1}$ or $-\sqrt{-1}$. We have from (4.6) that

$$
\begin{aligned}
& \left\{\begin{array}{l}
\lambda_{1}(\varsigma, x, y, z, w)=-\left(x+\varsigma^{3}(y-z)-w\right), \\
\lambda_{2}(\varsigma, x, y, z, w)=x+\varsigma^{3}(y-z)-w,
\end{array}\right. \\
& \left\{\begin{array}{l}
\lambda_{1}(\zeta, x, y, z, w)=x-\zeta^{3}(y+z)+w, \\
\lambda_{2}(\zeta, x, y, z, w)=x-\zeta^{3}(y+z)+w,
\end{array}\right. \\
& \left\{\begin{array}{l}
\lambda_{1}\left(A, x, y,-A x-A^{2} y, w\right)=-\left(A^{2} x+\left(A^{3}-A^{-1}\right) y-w\right), \\
\lambda_{2}\left(A, x, y,-A x-A^{2} y, w\right)=-\delta\left(A^{2} x+\left(A^{3}-A^{-1}\right) y-w\right),
\end{array}\right. \\
& \left\{\begin{array}{l}
\lambda_{1}\left(A, x,-A^{2} z-A w, z, w\right)=\delta\left(x-\left(A^{3}-A^{-1}\right) z-A^{2} w\right), \\
\lambda_{2}\left(A, x,-A^{2} z-A w, z, w\right)=x-\left(A^{3}-A^{-1}\right) z-A^{2} w
\end{array}\right.
\end{aligned}
$$

Given any ch-diagram $D$, we will denote by $D^{A}$ the diagram obtained from $D$ by splicing each classical crossing $Y$ to $)((A$-split $)$. For any state $\sigma$ of $D$ which assigns each marked vertex with $T_{\infty}$ or $T_{0}$, it is straightforward that $\left(D_{\sigma}\right)^{A}=$ $\left(D^{A}\right)_{\sigma}$, denoted simply by $D_{\sigma}^{A}$. Further, we denote the number of components of a classical link $K$ by $\|K\|$. 
Theorem 5.6. Let $D$ be any ch-diagram of a surface link $\mathcal{L}$ in $\mathbb{R}^{4}$. Then

$$
L_{D}(\varsigma, x, y, z, w)=\left(\varsigma^{3}\right)^{2|C(D)|-t(D)}(-1)^{\left\|D_{\sigma_{\infty}}^{A}\right\|+\left\|D_{\sigma_{0}}^{A}\right\|-t(D)-|V(D)|},
$$

where $\sigma_{\infty}$ and $\sigma_{0}$ are the states of $D$ which assign all marked vertices with $T_{\infty}$ and $T_{0}$, respectively.

Proof. From the observation (5.9), we obtain

$$
\lambda(\varsigma, x, y, z, w)=-\left(x+\varsigma^{3}(y-z)-w\right)^{2} .
$$

Let $\sigma$ be any state of $D$. Then $\left|C\left(D_{\sigma}\right)\right|=|C(D)|$ and it follows that

$$
\left\langle D_{\sigma}\right\rangle(\varsigma)=\left(\varsigma-\varsigma^{-1}\right)^{\left|C\left(D_{\sigma}\right)\right|}(-1)^{\left\|\left(D_{\sigma}\right)^{A}\right\|-1}=\left(\varsigma^{3}\right)^{|C(D)|}(-1)^{\left\|\left(D_{\sigma}\right)^{A}\right\|-1} .
$$

With this identity and (5.1) we obtain

$$
\begin{aligned}
{[[D]](\varsigma, x, y, z, w) } & =\sum_{\sigma \in \mathcal{S}_{\infty, 0}(D)} X^{\sigma(\infty)} Y^{\sigma(0)}\left\langle D_{\sigma}\right\rangle(\varsigma) \\
& =\sum_{\sigma \in \mathcal{S}_{\infty, 0}(D)} X^{\sigma(\infty)} Y^{\sigma(0)}\left(\varsigma^{3}\right)^{|C(D)|}(-1)^{\left\|\left(D_{\sigma}\right)^{A}\right\|-1} \\
& =\left(\varsigma^{3}\right)^{|C(D)|} \sum_{\sigma \in \mathcal{S}_{\infty, 0}(D)} X^{\sigma(\infty)} Y^{\sigma(0)}(-1)^{\left\|\left(D^{A}\right)_{\sigma}\right\|-1} \\
& =\left(\varsigma^{3}\right)^{|C(D)|}(X-Y)^{\left|V\left(D^{A}\right)\right|}(-1)^{\left\|\left(D^{A}\right)_{\sigma_{\infty}}\right\|-1} \\
& =\left(\varsigma^{3}\right)^{|C(D)|}\left(x+\varsigma^{3}(y-z)-w\right)^{|V(D)|}(-1)^{\left\|D_{\sigma_{\infty}}^{A}\right\|-1}, \\
{\left[\left[D^{*}\right]\right](\varsigma, x, y, z, w) } & =\sum_{\sigma \in \mathcal{S}_{\infty, 0}\left(D^{*}\right)} X^{\sigma(\infty)} Y^{\sigma(0)}\left\langle D_{\sigma}^{*}\right\rangle(\varsigma) \\
& =\sum_{\sigma \in \mathcal{S}_{\infty, 0}\left(D^{*}\right)} X^{\sigma(\infty)} Y^{\sigma(0)}\left(\varsigma^{3}\right)^{\left|C\left(D^{*}\right)\right|}(-1)^{\left\|\left(D_{\sigma}^{*}\right)^{A}\right\|-1} \\
& =\left(\varsigma^{3}\right)^{\left|C\left(D^{*}\right)\right|} \sum_{\sigma \in \mathcal{S}_{\infty, 0}\left(D^{*}\right)} X^{\sigma(\infty)} Y^{\sigma(0)}(-1)^{\left\|\left(D^{* A}\right)_{\sigma}\right\|-1} \\
& =\left(\varsigma^{3}\right)^{\left|C\left(D^{*}\right)\right|}(X-Y)^{\left|V\left(D^{* A}\right)\right|}(-1)^{\left\|\left(D^{* A}\right)_{\sigma_{\infty}}\right\|-1} \\
& =\left(\varsigma^{3}\right)^{|C(D)|}\left(x+\varsigma^{3}(y-z)-w\right)^{|V(D)|}(-1)^{\left\|\left(D^{A}\right)_{\sigma_{0}}\right\|-1},
\end{aligned}
$$

where $X=x+\varsigma y+\varsigma^{-1} z$ and $Y=\varsigma^{-1} y+\varsigma z+w$. Now, by Theorem 4.3 it follows that

$$
\begin{aligned}
L_{D}(\varsigma, x, y, z, w) & =\frac{\left(\varsigma^{3}\right)^{2|C(D)|}\left(x+\varsigma^{3}(y-z)-w\right)^{2|V(D)|}(-1)^{\left\|D_{\sigma_{\infty}}^{A}\right\|+\left\|D_{\sigma_{0}}^{A}\right\|-2}}{\left(-\varsigma^{3}\right)^{t(D)}\left(-\left(x+\varsigma^{3}(y-z)-w\right)^{2}\right)^{|V(D)|}} \\
& =\left(\varsigma^{3}\right)^{2|C(D)|-t(D)}(-1)^{\left\|D_{\sigma_{\infty}}^{A}\right\|+\left\|D_{\sigma_{0}}^{A}\right\|-t(D)-|V(D)|-2} .
\end{aligned}
$$

This completes the proof of Theorem 5.6 
On the other hand, one readily checks the conditions for (1) and (2) of Theorem 4.4. For $\epsilon= \pm 1$, it follows that

$$
\begin{aligned}
& \lambda_{2}(\zeta, x, y, z, w)=\lambda_{1}(\zeta, x, y, z, w)=x-\zeta^{3}(y+z)+w, \\
& \lambda_{2}\left(A, x, y,-A x-A^{2} y, w\right)=\lambda_{1}\left(A, x, y,-A x-A^{2} y, w\right) \neq 0 \Leftrightarrow A=\zeta ; \\
& \lambda_{1}\left(\zeta, x, y,-\zeta x-\zeta^{2} y, w\right)=-\left(\zeta^{2} x+\left(\zeta^{3}-\zeta^{-1}\right) y-w\right), \\
& \lambda_{2}\left(A, x,-A^{2} z-A w, z, w\right)=\lambda_{1}\left(A, x,-A^{2} z-A w, z, w\right) \neq 0 \Leftrightarrow A=\zeta ; \\
& \left.\lambda_{1}\left(\zeta, x,-\zeta^{2} z-\zeta w, z, w\right)=x-\left(\zeta^{3}-\zeta^{-1}\right) z-\zeta^{2} w\right), \\
& \lambda_{2}(\varsigma, x, y, z, w)=\lambda_{1}(\varsigma, x, y, z, w)^{-1} \Leftrightarrow \lambda_{1}(\varsigma, x, y, z, w)=\epsilon \sqrt{-1} \\
& \quad \Leftrightarrow w=w_{1}=x+\varsigma^{3}(y-z)+\epsilon \sqrt{-1} ; \\
& \lambda_{2}(\zeta, x, y, z, w)=\lambda_{1}(\zeta, x, y, z, w)^{-1} \Leftrightarrow \lambda_{1}(\zeta, x, y, z, w)=\epsilon \\
& \Leftrightarrow w=w_{2}=-x+\zeta^{3}(y+z)+\epsilon ; \\
& \lambda_{2}\left(A, x, y,-A x-A^{2} y, w\right)=\lambda_{1}\left(A, x, y,-A x-A^{2} y, w\right)^{-1} \\
& \Leftrightarrow \lambda_{1}\left(A, x, y,-A x-A^{2} y, w\right)=\frac{\epsilon}{\sqrt{\delta}} \\
& \Leftrightarrow w=w_{3}=A^{2} x+\left(A^{3}-A^{-1}\right) y+\frac{\epsilon}{\sqrt{\delta}} ; \\
& \lambda_{2}\left(A, x,-A^{2} z-A w, z, w\right)=\lambda_{1}\left(A, x,-A^{2} z-A w, z, w\right)^{-1} \\
& \Leftrightarrow \lambda_{1}\left(A, x,-A^{2} z-A w, z, w\right)=\epsilon \sqrt{\delta} \\
& \Leftrightarrow w=w_{4}=A^{-2} x-\left(A-A^{-3}\right) z-\frac{\epsilon}{A^{2} \sqrt{\delta}} .
\end{aligned}
$$

With these observations, we have

Theorem 5.7. Let $D$ be any ch-diagram of a surface link $\mathcal{L}$ in $\mathbb{R}^{4}$. Then

$$
J_{D}^{2}\left(\varsigma, x, y, z, w_{1}\right)=J_{D}^{2}\left(\varsigma, x, y, z, w_{1}\right)_{j}=(-1)^{\left\|D_{\sigma_{\infty}}^{A}\right\|-1}(\sqrt{-1})^{\eta_{j}(D)},
$$

where $\epsilon= \pm 1$ and $\sigma_{\infty}$ is the state of $D$ which assigns all marked vertices with $T_{\infty}$ and

$$
\begin{aligned}
& \eta_{1}(D)=|C(D)|+3|V(D)|-3 t_{+}(D)-e(D), \\
& \eta_{2}(D)=3|C(D)|+3|V(D)|-t_{+}(D)-e(D), \\
& \eta_{3}(D)=|C(D)|+|V(D)|-3 t_{+}(D)-3 e(D), \text { or } \\
& \eta_{4}(D)=3|C(D)|+|V(D)|-t_{+}(D)-3 e(D) .
\end{aligned}
$$

Proof. From (5.10), we obtain

$$
[[D]]\left(\varsigma, x, y, z, w_{1}\right)=\left(\varsigma^{3}\right)^{|C(D)|}(-\epsilon \sqrt{-1})^{|V(D)|}(-1)^{\left\|D_{\sigma_{\infty}}^{A}\right\|-1} .
$$

Hence, by Theorem 4.4 together with (5.11), it follows that

$$
\begin{aligned}
J_{D}^{2}\left(\varsigma, x, y, z, w_{1}\right) & =\left(-\varsigma^{3}\right)^{-t_{+}(D)}(\epsilon \sqrt{-1})^{-e(D)}[[D]]\left(\varsigma, x, y, z, w_{1}\right) \\
& =\left(\varsigma^{3}\right)^{|C(D)|}(-\epsilon \sqrt{-1})^{|V(D)|}(-1)^{\left\|D_{\sigma_{\infty}}^{A}\right\|-1}
\end{aligned}
$$


Thus

$$
\begin{aligned}
J_{D}^{2}(\mathbf{a}, \mathbf{s})= & \left(-\varsigma^{3}\right)^{-t_{+}(D)}(\epsilon \sqrt{-1})^{-e(D)}\left(\varsigma^{3}\right)^{|C(D)|}(-\epsilon \sqrt{-1})^{|V(D)|}(-1)^{\left\|D_{\sigma_{\infty}}^{A}\right\|-1} \\
= & \begin{cases}(-1)^{\left\|D_{\sigma_{\infty}}^{A}\right\|-1}(\sqrt{-1})^{\eta_{1}(D)} & \text { if } \epsilon=1, \varsigma^{3}=\sqrt{-1} \\
(-1)^{\left\|D_{\sigma_{\infty}}^{A}\right\|-1}(\sqrt{-1})^{\eta_{2}(D)} & \text { if } \epsilon=1, \varsigma^{3}=-\sqrt{-1} \\
(-1)^{\left\|D_{\sigma_{\infty}}^{A}\right\|-1}(\sqrt{-1})^{\eta_{3}(D)} & \text { if } \epsilon=-1, \varsigma^{3}=\sqrt{-1} \\
(-1)^{\left\|D_{\sigma_{\infty}}^{A}\right\|-1}(\sqrt{-1})^{\eta_{4}(D)} & \text { if } \epsilon=-1, \varsigma^{3}=-\sqrt{-1} .\end{cases}
\end{aligned}
$$

This completes the proof.

It should be noted that $J_{D}^{1}(\varsigma, x, y, z, w)$ cannot be defined because $\lambda_{2}(\varsigma, x, y, z, w)$ $=\lambda_{1}(\varsigma, x, y, z, w) \Leftrightarrow \lambda_{1}(\varsigma, x, y, z, w)=0$. On the other hand, for all $i=3,4,5,6$ and $A \neq 1, \lambda_{1}(\mathbf{a}, \mathbf{s})_{i} \neq \lambda_{2}(\mathbf{a}, \mathbf{s})_{i} \neq 0$. Hence for $i=3,4,5,6, J_{D}^{1}(\mathbf{a}, \mathbf{s})_{i}$ in Theorem 4.4(1) is not defined. For the remaining cases, by a slight modification of the proof of Theorems 5.6 and 5.7 above, one can obtain the following:

$$
\begin{aligned}
& L_{D}(\zeta, x, y, z, w)=\left(-\zeta^{3}\right)^{-t(D)}, \\
& L_{D}\left(A, x, y,-A x-A^{2} y, w\right)=\left(-A^{2}-A^{-2}\right)^{\mu_{-}(D)+\mu_{+}(D)-|V(D)|-2}, \\
& L_{D}\left(A, x,-A^{2} z-A w, z, w\right)=L_{D}\left(A, x, y,-A x-A^{2} y, w\right), \\
& J_{D}^{1}(\zeta, x, y, z, w)=\left(-\zeta^{3}\right)^{|C(D)|-t_{+}(D)}, \\
& J_{D}^{1}\left(\zeta, x, y,-\zeta x-\zeta^{2} y, w\right)=\left(-\zeta^{3}\right)^{t_{-}(D)-t_{+}(D)}, \\
& J_{D}^{1}\left(\zeta, x,-\zeta^{2} z-\zeta w, z, w\right)=1 \\
& J_{D}^{2}\left(\zeta, x, y, z, w_{2}\right)=\epsilon^{|V(D)|-e(D)} J_{D}^{1}(\zeta, x, y, z, w), \\
& J_{D}^{2}\left(A, x, y,-A x-A^{2} y, w_{3}\right)=\left(-A^{3}\right)^{t_{-}(D)-t_{+}(D)} \epsilon^{|V(D)|-e(D)} \delta^{\eta(D)}, \\
& J_{D}^{2}\left(A, x,-A^{2} z-A w, z, w_{4}\right)=\frac{J_{D}^{2}\left(A, x, y,-A x-A^{2} y, w_{3}\right)}{\left(-A^{3}\right)^{t_{-}(D)-t_{+}(D)}},
\end{aligned}
$$

where $\eta(D)=\frac{1}{2}\left(\mu_{+}(D)+\mu_{-}(D)-|V(D)|-2\right)$.

Remark 5.8. We remark that the invariants above are directly computed from a ch-diagram $D$ without computing the bracket polynomials of state diagrams. In the separate paper [17, we examine our method by using the number of components of classical links as its associated classical invariant and produce some invariants of surface links in 4-space.

Now we end this section with an example which gives the invariants of the fundamental surfaces in $\mathbb{R}^{4}$. For the sake of convenience, we shall denote the invariants by

$$
\begin{array}{ll}
L_{1}=L_{D}(\varsigma, x, y, z, w), & L_{6}^{j}=J_{D}^{2}\left(\varsigma, x, y, z, w_{1}\right)_{j}, j=1,2,3,4, \\
L_{2}=L_{D}(\zeta, x, y, z, w), & L_{7}=\left.J_{D}^{2}\left(\zeta, x, y, z, w_{2}\right)\right|_{\epsilon=-1}, \\
L_{3}=L_{D}\left(A, x, y,-A x-A^{2} y, w\right), & L_{8}=\left.J_{D}^{2}\left(A, x, y,-A x-A^{2} y, w_{3}\right)\right|_{\epsilon=-1}, \\
L_{4}=J_{D}^{1}(\zeta, x, y, z, w), & L_{9}=\left.J_{D}^{2}\left(A, x,-A^{2} z-A w, z, w_{4}\right)\right|_{\epsilon=-1} \\
L_{5}=J_{D}^{1}\left(\zeta, x, y,-\zeta x-\zeta^{2} y, w\right), &
\end{array}
$$


Example 5.9. Let $D_{1}, D_{2}$ and $D_{3}$ denote the ch-diagrams of $0_{1}, 2_{1}^{-1}$ and $2_{1}^{1}$ in Yoshikawa's table, respectively, and denote $I=\sqrt{-1}$. Then

\begin{tabular}{|l|rrrrrrrrrrrr|}
\hline & $L_{1}$ & $L_{2}$ & $L_{3}$ & $L_{4}$ & $L_{5}$ & $L_{6}^{1}$ & $L_{6}^{2}$ & $L_{6}^{3}$ & $L_{6}^{4}$ & $L_{7}$ & $L_{8}$ & $L_{9}$ \\
\hline $0_{1}$ & 1 & 1 & 1 & 1 & 1 & 1 & 1 & 1 & 1 & 1 & 1 & 1 \\
$2_{1}^{-1}$ & -1 & 1 & $\frac{1}{\delta}$ & 1 & 1 & $-I$ & $-I$ & $I$ & $I$ & -1 & $\frac{-1}{A^{6} \sqrt{\delta}}$ & $\frac{-1}{\sqrt{\delta}}$ \\
$2_{1}^{1}$ & 1 & 1 & $\frac{1}{\delta^{2}}$ & 1 & 1 & -1 & -1 & -1 & -1 & 1 & $\frac{1}{\delta}$ & $\frac{1}{\delta}$ \\
\hline
\end{tabular}

\section{REFERENCES}

1. M. Asada, An unknotting sequence for surface-knots represented by ch-diagrams and their genera, Kobe J. Math. 18 (2001), 163-180. MR.1907671 (2003d:57049)

2. J.S. Carter, J.H. Rieger, M. Saito, A combinatorial description of knotted surfaces and their isotopies, Adv. Math. 127(1997), 1-51. MR1445361(98c:57023)

3. J.S. Carter, D. Jelsovsky, S. Kamada, L. Langford, M. Saito, Quandle cohomology and statesum invariants of knotted curves and surfaces, Trans. Amer. Math. Soc. 355 (2003), 39473989. MR 1990571 (2005b:57048)

4. D. Cox, J. Little, D. O'Shea, Ideals, Varieties, and Algorithms, Springer, 1997. MR 1417938 (97h:13024)

5. J.S. Carter, M. Saito, Knotted surfaces and their diagrams, Mathematical surveys and monographs 55, Amer. Math. Soc., Providence, RI, 1998. MR1487374 (98m:57027)

6. R.H. Fox, A quick trip through knot theory, in Toplogy of 3-manifolds and Related Topics, Prentice-Hall, Inc., Englewood Cliffs, N.J.(1962), 120-167. MR0140099 (25:3522)

7. R.H. Fox and J.W. Milnor, Singularities of 2-spheres in 4-space and equivalence of knots, (unpublished version).

8. S. Kamada, Non-orientable surfaces in 4-space, Osaka J. Math. 26(1989), 367-385. MR.1017592 (91g:57022)

9. S. Kamada, Surfaces in $\mathbb{R}^{4}$ of braid index three are ribbon, J. Knot Theory Ramifications 1(1992), 137-160. MR.1164113 (93h:57039)

10. S. Kamada, A characterization of groups of closed orientable surfaces in 4-space, Topology 33(1994), 113-122. MR.1259518 (95a:57002)

11. S. Kamada, Braid and Knot Theory in Dimension Four, Mathematical Surveys and Monographs 95(2002), American Mathematical Society. MR1900979 (2003d:57050)

12. S. Kamada, in preparation.

13. L.H. Kauffman, State models and the Jones polynomial, Topology 26(1987), 395-407. MR.899057 (88f:57006)

14. A. Kawauchi, T. Shibuya, S. Suzuki, Descriptions on surfaces in four-space, I; Normal forms, Math. Sem. Notes Kobe Univ. 10(1982), 75-125. MR672939 (84d:57017)

15. A. Kawauchi, A survey of knot theory, Birkhäuser, 1996. MR1417494 (97k:57011)

16. S. Y. Lee, Invariants of surface links in $\mathbb{R}^{4}$ via skein relation, J. Knot Theory Ramifications 17(2008), 439-469.

17. S. Y. Lee, Invariants of surfaces in 4-space via an elementary classical link invariant, preprint available at http://home.pusan.ac.kr/ $\sim$ knot.

18. S. Y. Lee, Invariants of oriented surfaces in 4 -space via invariants for magnetic graphs, in preparation.

19. L.Tr. Lomonaco, The homotopy groups of knots I. How to compute the algebraic 2-type, Pacific J. Math. 95(1981), 349-390. MR632192 (83a:57025)

20. Y. Miyazawa, Magnetic graphs and an invariant for virtual links, J. Knot Theory Ramifications 15 (2006), 1319-1334. MR.2286126 (2007k:57014)

21. Y. Miyazawa, A multi-variable polynomial invariant for virtual knots and links, to appear in J. Knot Theory Ramifications.

22. Y. Nakanishi, M. Teragaito, 2-knots from a view of moving picture, Kobe J. Math. 8(1991), 161-172. MR 1159867 (94d:57052)

23. D. Roseman, Reidemeister-type moves for surfaces in four-dimensional space, Knot theory (Warsaw, 1995), Polish Acad. Sci.(1998), 347-380. MR.1634466 (99f:57029)

24. L. Rudolph, Braided surfaces and Seifert ribbons for closed braids, Comm. Math. Helv. 58(1983), 1-37. MR699004 (84j:57006) 
25. M. Soma, Surface-links with square-type ch-graphs, Proceedings of the First Joint JapanMexico Meeting in Topology (Morelia, 1999), Topology Appl. 121 (2002), 231-246. MR.1903693 (2003c:57024)

26. F.J. Swenton, On a calculus for 2-knots and surfaces in 4-space, J. Knot Theory Ramifications 10(2001), 1133-1141. MR1871221 (2002j:57043)

27. K. Yoshikawa, An enumeration of surfaces in four-space, Osaka J. Math. 31(1994), 497-522. MR.1309400 (95m:57037)

Department of Mathematics, Pusan National University, Pusan 609-735, Korea

E-mail address: sangyoul@pusan.ac.kr 